\title{
Existence and Performance of Shalvi-Weinstein Estimators
}

\author{
Philip Schniter, Member, IEEE, and Lang Tong, Member, IEEE
}

\begin{abstract}
The Shalvi-Weinstein (SW) criterion has become popular in the design of blind linear estimators of i.i.d. processes transmitted through unknown linear channels in the presence of unknown additive interference. Here, we analyze SW estimators in a general multiple-input multiple-output (MIMO) setting that allows near-arbitrary source/interference distributions and noisy noninvertible channels. The main contributions of this paper are i) simple tests for the existence of $\mathrm{SW}$ estimators for the desired source and ii) bounding expressions for the MSE of SW estimators that are a function of the minimum attainable MSE and the kurtoses of the source and interferers.
\end{abstract}

Index Terms-Blind beamforming, blind deconvolution, blind equalization, blind source separation, Shalvi-Weinstein algorithm.

\section{INTRODUCTION}

C ONSIDER the linear estimation problem of Fig. 1, where a desired source sequence $\left\{s_{n}^{(0)}\right\}$ combines linearly with $K$ sources of interference $\left\{s_{n}^{(k)}\right\}$ through vector channels $\left\{\mathbf{h}^{(0)}(z), \ldots, \mathbf{h}^{(K)}(z)\right\}$. Our goal is to estimate the desired source using the (vector) linear estimator $\mathbf{f}(z)$. The linear estimates $\left\{y_{n}\right\}$ that minimize the mean-squared error (MSE)

$$
J_{\mathrm{m}}\left(y_{n}\right):=\mathrm{E}\left\{\left|y_{n}-s_{n-\nu}^{(0)}\right|^{2}\right\}
$$

are generated by the minimum MSE (MMSE) estimator, or Wiener estimator $\mathbf{f}_{\mathrm{m}}(z)$. Specification of $\mathbf{f}_{\mathrm{m}}(z)$, however, requires knowledge of the joint statistics of the observed sequence $\left\{\mathbf{r}_{n}\right\}$ and the desired source sequence $\left\{s_{n}^{(0)}\right\}$, which are typically unavailable when the channel is unknown.

When only the statistics of the observed sequence $\left\{\mathbf{r}_{n}\right\}$ are known, it may still be possible to estimate $\left\{s_{n}^{(0)}\right\}$ up to unknown magnitude and delay, i.e., $y_{n}=\sum_{i} \mathbf{f}_{i}^{\mathrm{H}} \mathbf{r}_{n-i} \approx \alpha s_{n-\nu}^{(0)}$ for some $\alpha \in \mathbb{C}$, some $\nu \in \mathbb{Z}$, and all $n$. The literature refers to this problem as blind estimation (or blind deconvolution).

In [1], Shalvi and Weinstein showed that for i.i.d. sources, noiseless invertible channels, and adequately parameterized estimators, perfect blind estimation is possible with knowledge of only the second- and fourth-order moments of the estimates

Manuscript received April 21, 2000; revised May 11, 2001. The associate editor coordinating the review of this paper and approving it for publication was Prof. Colin F. Cowan.

P. Schniter is with the Department of Electrical Engineering, The Ohio State University, Columbus OH 43210 USA (e-mail: schniter@ee.eng.ohiostate.edu).

L. Tong is with the Department of Electrical Engineering, Cornell University, Ithaca NY 14853 USA (e-mail: ltong@ee.cornell.edu).

Publisher Item Identifier S 1053-587X(01)07055-6.

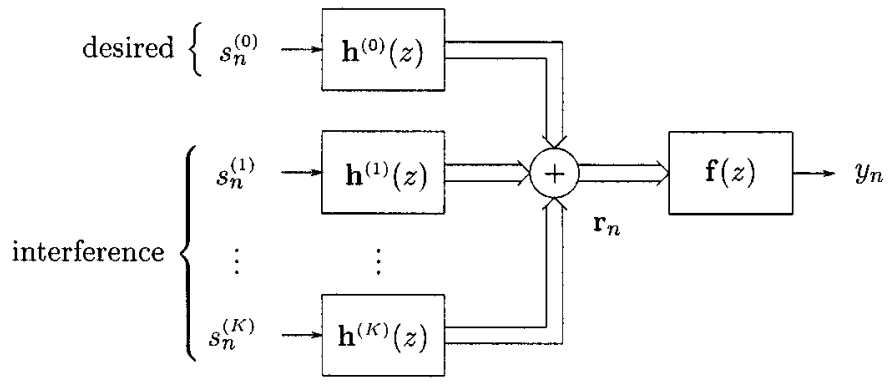

Fig. 1. Linear system model with $K$ sources of interference.

$\left\{y_{n}\right\}$. Based on this observation, they proposed a blind estimation scheme that manipulates $\mathrm{f}(z)$ to maximize the absolute kurtosis of the estimates $\left\{y_{n}\right\}$ subject to a fixed variance, i.e.,

$$
\max \left|\mathcal{K}_{y}\right| \text { s.t. } \sigma_{y}^{2}=1
$$

where, for zero-mean $\left\{y_{n}\right\}$, we have $\sigma_{y}^{2}=\mathrm{E}\left\{\left|y_{n}\right|^{2}\right\}$ and kurtosis

$$
\mathcal{K}_{y}:=\mathrm{E}\left\{\left|y_{n}\right|^{4}\right\}-2 \mathrm{E}^{2}\left\{\left|y_{n}\right|^{2}\right\}-\left|\mathrm{E}\left\{y_{n}^{2}\right\}\right|^{2} .
$$

Although, in name, Shalvi and Weinstein have received credit for the criterion of (2), it has a history that long predates their publication [1]. In fact, the use of kurtosis as a blind estimation criterion can be traced back to the early 1950s [2]. During the late 1970s, Wiggins [3] successfully applied the kurtosis criterion to problems in geophysical exploration, the results of which prompted a more detailed analysis by Donoho in 1981 [4].

As proven independently in [1] and [4], unconstrained linear estimators locally maximizing the SW criterion yield perfect blind estimates of a single non-Gaussian i.i.d. source transmitted through a noiseless invertible linear channel. In practical situations, however, we expect inadequately parameterized estimators, noninvertible channels, as well as noise and/or interference of a potentially non-Gaussian nature. Are Shalvi-Weinstein (SW) estimators useful in these cases? How do SW estimates compare with optimal (linear) estimators, say, in a meansquared sense?

For a finite impulse response (FIR) estimator and noiseless causal bounded-input bounded-output (BIBO) stable channels, Regalia and Mboup studied various properties of SW minimizers [5]. Although they provided evidence that the SW and MMSE estimators are closely related in many cases, their approach did not lead to upper bounds on the performance of the SW estimator.

Recently, Feng and Chi studied the properties of unconstrained infinite-dimensional SW estimators of a non-Gaussian 
source in the presence of Gaussian noise [6], [7]. Using a frequency-domain approach, they observed relationships between the Weiner and SW estimators that bear similarity ${ }^{1}$ to the time-domain relationships derived previously by Regalia and Mboup. The complexity of the analytical relationships derived by Feng and Chi prevents their translation into meaningful statements about the MSE performance of SW estimators, however.

In this paper, we study the performance of constrained FIR SW estimators under the assumptions of the model in Section II-A: desired source with arbitrary non-Gaussian distribution, noise and interference with arbitrary distribution, and causal BIBO stable channels. The main contributions of this paper are i) simple tests for the existence of SW estimators for the desired source and ii) bounding expressions for the MSE of SW estimators that are a function of the minimum MSE attainable under the same conditions. These bounds, which are derived under the multisource linear model of Fig. 1, provide a formal link between the SW and Wiener estimators in a very general context.

The organization of the paper is as follows. Section II discusses the properties of the system model and MSE estimation criteria in detail, Section III derives the bound for the MSE performance of the SW criterion, and Section IV presents the results of numerical simulations demonstrating the efficacy of our bounding technique. Section V concludes the paper.

\section{BACKGROUND}

In this section, we give more detailed information on the linear system model and the MSE, unbiased MSE, and SW criteria. The following notation is used throughout.

$(\cdot)^{\mathrm{t}}$ transpose;

$(\cdot)^{*}$ conjugate;

$(\cdot)^{\mathrm{H}}$ Hermitian;

$(\cdot)^{\dagger}$ Moore-Penrose pseudo-inverse.

Likewise, $\mathrm{E}\{\cdot\}$ denotes expectation, $\|\mathrm{x}\|_{p}$ the $p$-norm defined by $\sqrt[p]{\sum_{i}\left|x_{i}\right|^{p}}$, and $\mathbb{R}^{+}$the field of nonnegative real numbers. In general, we use boldface lowercase type to denote vector quantities and boldface uppercase type to denote matrix quantities.

\section{A. Linear System Model}

First, we formalize the linear time-invariant multichannel model illustrated in Fig. 1. Say that the desired symbol sequence $\left\{s_{n}^{(0)}\right\}$ and $K$ sources of interference $\left\{s_{n}^{(1)}\right\}, \ldots,\left\{s_{n}^{(K)}\right\}$ each pass through separate linear "channels" before being observed at the receiver. The interference processes may correspond, e.g., to interference signals or additive noise processes. ${ }^{2}$ In addition, say that the receiver uses a sequence of $P$-dimensional vector observations $\left\{\mathbf{r}_{n}\right\}$ to estimate (a possibly delayed version of) the desired source sequence, where the case $P>1$ corresponds to a receiver that employs multiple sensors

\footnotetext{
${ }^{1}$ Keep in mind that Regalia and Mboup studied constrained estimators in noiseless settings, whereas Feng and Chi studied unconstrained estimators in noisy settings.

${ }^{2}$ To model AWGN of variance $\sigma_{w}^{2}$ at $P$ sensors, dedicate $P$ Gaussian sources $\left\{s_{n z}^{(k)}\right\}$ with corresponding channels of the form $\mathbf{h}^{(k)}(z)=$ $\left(0, \ldots, 0, \sigma_{w} / \sigma_{s}, 0, \ldots, 0\right)^{\mathrm{t}}$.
}

and/or samples at an integer multiple of the symbol rate. The observations $\mathbf{r}_{n}$ can be written $\mathbf{r}_{n}=\sum_{k=0}^{K} \sum_{i=0}^{\infty} \mathbf{h}_{i}^{(k)} s_{n-i}^{(k)}$, where $\left\{\mathbf{h}_{n}^{(k)}\right\}$ denote the impulse response coefficients of the linear time-invariant (LTI) channel $\mathbf{h}^{(k)}(z)$, which is assumed to be causal BIBO stable. Note that such $\mathbf{h}^{(k)}(z)$ admit infinite impulse response (IIR) channel models.

From the vector-valued observation sequence $\left\{\mathbf{r}_{n}\right\}$, the receiver generates a sequence of linear estimates $\left\{y_{n}\right\}$ of $\left\{s_{n-\nu}^{(0)}\right\}$, where $\nu$ is a fixed integer. Using $\left\{\mathrm{f}_{n}\right\}$ to denote the impulse response of the linear estimator $\mathrm{f}(z)$, the estimates are formed as $y_{n}=\sum_{i=-\infty}^{\infty} \mathbf{f}_{i}^{\mathrm{H}} \mathrm{r}_{n-i}$. We will assume that the linear system $\mathbf{f}(z)$ is constrained FIR, i.e., certain coefficients of $\mathbf{f}(z)$ may be held at zero.

In the sequel, we will focus almost exclusively on the global channel-plus-estimator response $q^{(k)}(z):=\mathrm{f}^{\mathrm{H}}(z) \mathbf{h}^{(k)}(z)$. The impulse response coefficients of $q^{(k)}(z)$ can be written

$$
q_{n}^{(k)}=\sum_{i} \mathrm{f}_{i}^{\mathrm{H}} \mathbf{h}_{n-i}^{(k)}
$$

allowing the estimates to be written as $y_{n}=\sum_{k=0}^{K} \sum_{i} q_{i}^{(k)}$ $s_{n-i}^{(k)}$. Adopting the following vector notation helps to streamline the remainder of the paper.

$$
\begin{aligned}
\underline{\boldsymbol{q}}^{(k)}:= & \left(\ldots, q_{-1}^{(k)}, q_{0}^{(k)}, q_{1}^{(k)}, \ldots\right)^{\mathrm{t}} \\
\underline{\boldsymbol{q}}:= & \left(\ldots, q_{-1}^{(0)}, q_{-1}^{(1)}, \ldots, q_{-1}^{(K)}, q_{0}^{(0)}, q_{0}^{(1)}, \ldots\right. \\
& \left.q_{0}^{(K)}, q_{1}^{(0)}, q_{1}^{(1)}, \ldots, q_{1}^{(K)}, \ldots\right)^{\mathrm{t}} \\
\underline{\boldsymbol{s}}^{(k)}(n):= & \left(\ldots, s_{n+1}^{(k)}, s_{n}^{(k)}, s_{n-1}^{(k)}, \ldots\right)^{\mathrm{t}} \\
\underline{\boldsymbol{s}}(n):= & \left(\ldots, s_{n+1}^{(0)}, s_{n+1}^{(1)}, \ldots, s_{n+1}^{(K)}, s_{n}^{(0)}, s_{n}^{(1)}, \ldots\right. \\
& \left.s_{n}^{(K)}, s_{n-1}^{(0)}, s_{n-1}^{(1)}, \ldots, s_{n-1}^{(K)}, \ldots\right)^{\mathrm{t}} .
\end{aligned}
$$

For instance, the estimates can be rewritten concisely as

$$
y_{n}=\sum_{k=0}^{K} \underline{\boldsymbol{q}}^{(k) \mathrm{t}} \underline{\boldsymbol{s}}^{(k)}(n)=\underline{\boldsymbol{q}}^{\mathrm{t}} \underline{\boldsymbol{s}}(n) .
$$

The source-specific unit vector $\underline{e}_{\nu}^{(k)}$ will also prove convenient. $\underline{\boldsymbol{e}}_{\nu}^{(k)}$ is a column vector with a single nonzero element of value 1 located such that $\boldsymbol{q}^{\dagger} \underline{\boldsymbol{e}}_{\nu}^{(k)}=q_{\nu}^{(k)}$.

We now point out a few important properties of $\underline{\boldsymbol{q}}$. First, it is important to recognize that a particular channel and set of estimator constraints will restrict the set of attainable global responses, which we denote by $\mathcal{Q}_{\mathrm{a}}$. For example, when the estimator is unconstrained FIR, (4) implies that $\underline{\boldsymbol{q}} \in \mathcal{Q}_{\mathrm{a}}=\operatorname{row}(\boldsymbol{H})$, where

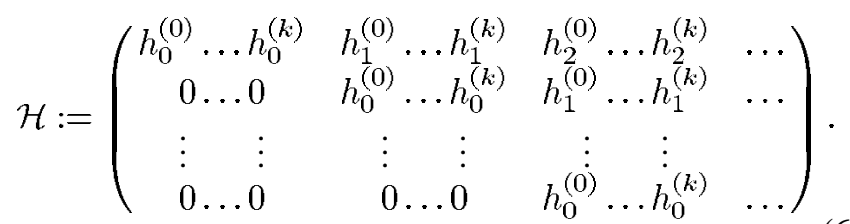


Restricting the estimator to be sparse would generate different attainable sets $\mathcal{Q}_{\mathrm{a}}$. Next, BIBO stable $\mathbf{f}(z)$ and $\mathbf{h}^{(k)}(z)$ imply BIBO stable $q^{(k)}(z)$ so that $\left\|\underline{q}^{(k)}\right\|_{p}$ exists for all $p \geq 1$, and thus, $\|\underline{\underline{q}}\|_{p}$ does as well. Finally, the fact that $\underline{\boldsymbol{q}}$ is generated from finite linear combinations of the rows of $\mathcal{H}$ implies that $\boldsymbol{q}$ lies in a finite-dimensional subspace of $\ell_{2}$.

Throughout the paper, we make the following assumptions on the $K+1$ source processes.

S1) For all $k,\left\{s_{n}^{(k)}\right\}$ is zero-mean i.i.d.

S2) $\quad\left\{s_{n}^{(0)}\right\}, \ldots,\left\{s_{n}^{(K)}\right\}$ are jointly statistically independent.

S3) For all $k, \mathrm{E}\left\{\left|s_{n}^{(k)}\right|^{2}\right\}=\sigma_{s}^{2}$.

S4) $\mathcal{K}_{s}^{(0)} \neq 0$.

S5) If, for any $k, q^{(k)}(z)$ or $\left\{s_{n}^{(k)}\right\}$ is not real-valued, then $\left.\mathrm{E}\left\{s_{n}^{(k)}\right)^{2}\right\}=0$ for all $k$.

\section{B. Mean-Squared Error Criterion}

The well-known mean-squared error (MSE) criterion was defined in (1) in terms of the estimate $y_{n}$ and the estimand $s_{n-\nu}^{(0)}$. Using S1)-S3), we may rewrite (1) in terms of the global response $\underline{q}$ :

$$
J_{\mathrm{m}}(\underline{\boldsymbol{q}})=\left\|\underline{\boldsymbol{q}}-\underline{\boldsymbol{e}}_{\nu}^{(0)}\right\|_{2}^{2} \sigma_{s}^{2} .
$$

Denoting MMSE quantities by the subscript " $m$," it is well known that, for FIR channels, S1)-S3) imply an MMSE channel-plus-estimator of the form $\underline{\boldsymbol{q}}_{\mathrm{m}}=\boldsymbol{\mathcal { H }}^{\mathrm{t}}\left(\mathcal{H}^{*} \boldsymbol{H}^{\mathrm{t}}\right)^{\dagger} \boldsymbol{\mathcal { H }}^{*} \underline{\boldsymbol{e}}_{\nu}^{(0)}$. A similar expression can be derived for the IIR case. (For derivations, see [8].)

\section{Unbiased Mean-Squared Error}

Since both symbol power and channel gain are unknown in the "blind" scenario, blind estimators suffer from a gain ambiguity. To ensure that our estimator performance evaluation is meaningful in the face of such ambiguity, we base our evaluation on normalized versions of the blind estimators and normalize by the receiver gain $q_{\nu}^{(0)}$. Given that the estimate $y_{n}$ can be decomposed into signal and interference terms as

$$
y_{n}=q_{\nu}^{(0)} s_{n-\nu}^{(0)}+\overline{\boldsymbol{q}}^{\mathrm{t}} \overline{\boldsymbol{s}}(n)
$$

where $\bar{q}$ denotes $\boldsymbol{q}$ with the $q_{\nu}^{(0)}$ term removed and $\bar{s}(n)$ denotes $\underline{s}(n)$ with the $s_{n-\nu}^{(0)}$ term removed, the normalized estimate $y_{n} / q_{\nu}^{(0)}$ can be referred to as "conditionally unbiased" since $\mathrm{E}\left\{y_{n} / q_{\nu}^{(0)} \mid s_{n-\nu}^{(0)}\right\}=s_{n-\nu}^{(0)}$.

The (conditionally) unbiased MSE (UMSE) associated with $y_{n}$, which is an estimate of $s_{n-\nu}^{(0)}$, is then defined

$$
J_{\mathbf{u}}\left(y_{n}\right):=E\left\{\left|\frac{y_{n}}{q_{\nu}^{(0)}}-s_{n-\nu}^{(0)}\right|^{2}\right\} .
$$

Substituting (8) into (9), we find that

$$
J_{\mathbf{u}}\left(q_{\nu}^{(0)}, \overline{\boldsymbol{q}}\right)=\frac{E\left\{\left|\overline{\boldsymbol{q}}^{\mathrm{t}} \overline{\boldsymbol{s}}(n)\right|^{2}\right\}}{\left|q_{\nu}^{(0)}\right|^{2}}=\frac{\|\left.\overline{\boldsymbol{q}}\right|_{2} ^{2}}{\left|q_{\nu}^{(0)}\right|^{2}} \sigma_{s}^{2}
$$

where the second equality invokes assumptions S1)-S3).
The UMSE criterion is similar to the popular "intersymbol interference measure" that appears in, e.g., [1]. Note, however, that the UMSE criterion measures the effects of additive noise in addition to co-channel interference and ISI.

\section{SW Performance Under General AdDitive INTERFERENCE}

In this section, we derive tight bounds for the UMSE of SW symbol estimators that

i) have a closed-form expression;

ii) support arbitrary additive noise and interference;

iii) support complex-valued channels and estimators;

iv) support IIR (as well as FIR) channels.

Sections III-A and B outline our approach, Section III-C presents the main results, and Section III-D comments on these results. Proof details appear in the Appendix.

\section{A. Bounding Strategy}

Since $y_{n}=\underline{\boldsymbol{q}}^{\mathrm{t}} \underline{\boldsymbol{s}}(n)$ for $\underline{\boldsymbol{q}} \in \mathcal{Q}_{\mathrm{a}}$, source assumptions S1)-S5) imply that [9]

$$
\begin{aligned}
& \mathcal{K}_{y}=\sum_{k}\left\|\underline{\boldsymbol{q}}^{(k)}\right\|_{4}^{4} \mathcal{K}_{s}^{(k)} \\
& \sigma_{y}^{2}=\|\underline{q}\|_{2}^{2} \sigma_{s}^{2} .
\end{aligned}
$$

This allows us to rewrite the SW criterion (2) as

$$
\max _{\underline{q} \in \mathcal{Q}_{\mathrm{a}} \cap \mathcal{S}}\left|\sum_{k}\left\|\underline{q}^{(k)}\right\|_{4}^{4} \mathcal{K}_{s}^{(k)}\right|
$$

where $\mathcal{S}$ denotes the unit sphere $\mathcal{S}:=\left\{\underline{\boldsymbol{q}}\right.$ s.t. $\left.\|\underline{\boldsymbol{q}}\|_{2}=1\right\}$.

Although the SW criterion admits multiple solutions, we are only interested in those that correspond to the estimation of the zeroth user's symbols at delay $\nu$. Thus, the set of SW global responses associated with the $\{0, \nu\}$ pair is defined by the local maxima

$$
\left\{\underline{\boldsymbol{q}}_{\mathrm{sw}}\right\}:=\left\{\arg \max _{\underline{q} \in \mathcal{Q}_{\mathrm{a}} \cap \mathcal{S}}\left|\sum_{k}\left\|\underline{\boldsymbol{q}}^{(k)}\right\|_{4}^{4} \mathcal{K}_{s}^{(k)}\right|\right\} \cap \mathcal{C}_{\nu}^{(0)}
$$

where $\mathcal{C}_{\nu}^{(0)}$ specifies the "dominant cone" associated with the user/delay pair $\{0, \nu\}$ :

$$
\mathcal{C}_{\nu}^{(0)}:=\left\{\underline{\boldsymbol{q}} \text { s.t. }\left|q_{\nu}^{(0)}\right|>\max _{(k, \delta) \neq(0, \nu)}\left|q_{\delta}^{(k)}\right|\right\} .
$$

It is not possible to write general closed-form expressions for $\left\{\underline{\boldsymbol{q}}_{\mathrm{sw}}\right\}$, making it difficult to characterize SW performance. In fact, $\left\{\underline{\boldsymbol{q}}_{\mathrm{sw}}\right\}$ may be empty, although for now, we assume that this is not the case.

It is, however, possible to say something about the location of $\underline{\boldsymbol{q}}_{\mathrm{sw}}$. Specifically, we know that within $\mathcal{Q}_{\mathrm{a}} \cap \mathcal{S}$, there exist neighborhoods of $\underline{q}_{\mathrm{sw}}$ generating strictly lower $\left|\mathcal{K}_{y}\right|$ than that characterizing the local $\left|\mathcal{K}_{y}\right|$-maximizer $\underline{\boldsymbol{q}}_{\mathrm{sw}}$. One such neighborhood can be constructed as follows. Given a reference response $\underline{\boldsymbol{q}}_{\mathrm{r}} \in \mathcal{Q}_{\mathrm{a}} \cap \mathcal{S} \cap \mathcal{C}_{\nu}^{(0)}$ generating estimates with reference kurtosis ${ }_{\mathrm{K}}^{\mathcal{K}}$, a neighborhood containing $\underline{\boldsymbol{q}}_{\mathrm{sw}}$ is formed by

$$
\mathcal{Q}_{\left|\mathcal{K}_{y}\right|>\left|\mathcal{K}_{\mathbf{r}}\right|}:=\left\{\underline{\boldsymbol{q}} \text { s.t. }\left|\sum_{k}\left\|\underline{\boldsymbol{q}}^{(k)}\right\|_{4}^{4} \mathcal{K}_{s}^{(k)}\right|>\left|\mathcal{K}_{\mathrm{r}}\right|\right\} \cap \mathcal{C}_{\nu}^{(0)} \cap \mathcal{S}
$$

as long as the boundary (hence, closure) of $\mathcal{Q}_{\left|\mathcal{K}_{y}\right|>\left|\mathcal{K}_{\mathrm{r}}\right|}$ lies entirely within $\mathcal{C}_{\nu}^{(0)} \cap \mathcal{S}$. This claim is supported by the Weierstrass 


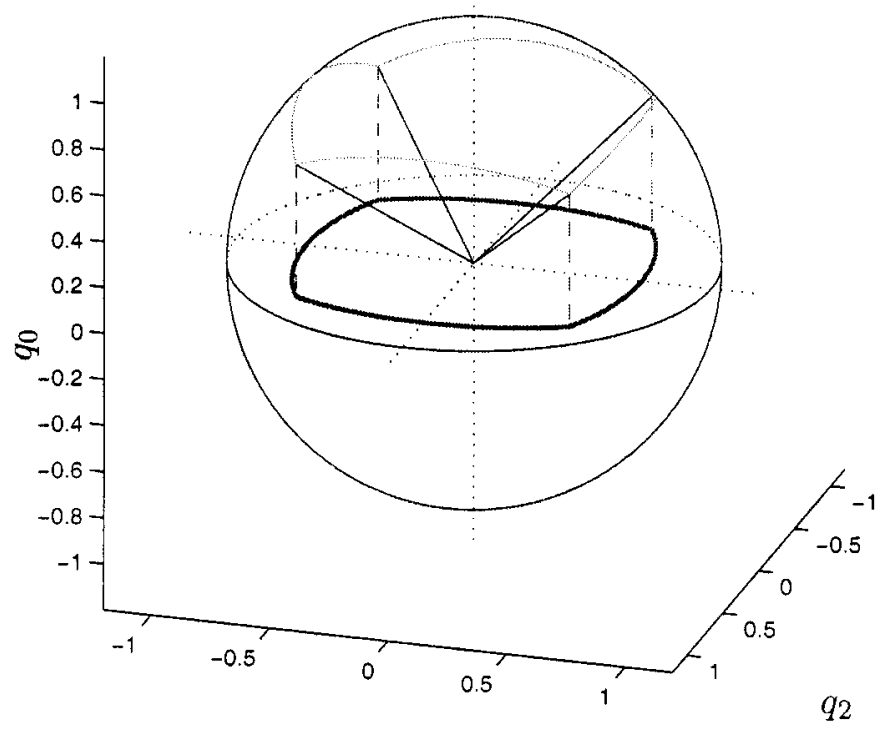

$q_{1}$

Fig. 2. Thick arcs demarcate the boundary of $\overline{\mathcal{Q}}_{C} \cap \mathcal{S}$ on the horizontal plane and thin arcs the boundary of $\mathcal{C}_{\nu}^{(0)} \cap \mathcal{S}$ on the sphere for $\boldsymbol{q} \in \mathbb{R}^{3}$.

theorem [10, p. 40], which says that a continuous cost functional must have a local maximum on a compact set if there exist points in the interior that give cost strictly higher than anywhere on the boundary. Note that the boundary of $\mathcal{Q}_{\left|\mathcal{K}_{y}\right|>\left|\mathcal{K}_{\mathrm{r}}\right|}$ is essentially a "level set" of cost functional $\left|\mathcal{K}_{y}\right|$ within $\mathcal{C}_{\nu}^{(0)} \cap \mathcal{S}$. bound

When $\underline{\boldsymbol{q}}_{\mathrm{sw}} \in \mathcal{Q}_{\left|\mathcal{K}_{y}\right|>\left|\mathcal{K}_{\mathrm{r}}\right|}$, we have the SW-UMSE upper

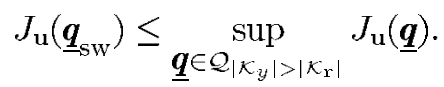

The choice of reference $\underline{q}_{r}$ is important since it affects the tightness (and existence) of bound (14). We will eventually choose the scaled MMSE reference $\underline{\boldsymbol{q}}_{\mathrm{r}}=\underline{\boldsymbol{q}}_{\mathrm{m}} /\left\|\underline{\boldsymbol{q}}_{\mathrm{m}}\right\|_{2}$ (when $\underline{\boldsymbol{q}}_{\mathrm{m}} \in \mathcal{C}_{\nu}^{(0)}$ ) since it is an established benchmark with a closed-form expression, and we will derive conditions that ensure that $\underline{\boldsymbol{q}}_{\mathrm{m}}$ can be used to specify a valid local neighborhood of $\underline{\boldsymbol{q}}_{\mathrm{sw}}$.

\section{B. Bound Evaluation in Interference Space}

Equation (14) requires maximization over a region of the unit sphere in $\boldsymbol{q}$-space that would be cumbersome if attempted directly. It will be more convenient to reparameterize our quantities in the interference $(\overline{\boldsymbol{q}})$ space, as explained below. Since both the SW and UMSE criteria are invariant to phase rotation of $\underline{q}$ (i.e., scalar multiplication of $\underline{q}$ by $e^{j \phi}$ for $\phi \in \mathbb{R}$ ), we can, w.l.o.g., restrict the our attention to the set of "de-rotated" global responses $\left\{\underline{\boldsymbol{q}}\right.$ s.t. $\left.q_{\nu}^{(0)} \in \mathbb{R}^{+}\right\}$. For derotated responses $\underline{\boldsymbol{q}} \in \mathcal{S} \cap \mathcal{C}_{\nu}^{(0)}$, we know $q_{\nu}^{(0)}=\sqrt{1-\|\overline{\boldsymbol{q}}\|_{2}^{2}}$, which implies that such $\underline{\boldsymbol{q}}$ are completely described by their interference response $\overline{\boldsymbol{q}}$ (which were defined in Section II-C). Moreover, these interference responses lie within the bounded region $\overline{\mathcal{Q}}_{\mathcal{C} \cap \mathcal{S}}$

$$
\overline{\mathcal{Q}}_{\mathcal{C} \cap \mathcal{S}}:=\left\{\overline{\boldsymbol{q}} \text { s.t. } \sqrt{1-\|\overline{\boldsymbol{q}}\|_{2}^{2}}>\max _{(k, \delta) \neq(0, \nu)}\left|q_{\delta}^{(k)}\right|\right\} .
$$

Essentially, $\overline{\mathcal{Q}}_{\mathcal{C} \cap \mathcal{S}}$ is a projection of $\mathcal{C}_{\nu}^{(0)} \cap \mathcal{S}$ onto the interference space, as illustrated in Fig. 2. Expressed in terms of the interference response, (10) and (11) become

$$
\begin{aligned}
\left.\frac{J_{\mathbf{u}}\left(q_{\nu}^{(0)}, \overline{\boldsymbol{q}}\right)}{\sigma_{s}^{2}}\right|_{\underline{\boldsymbol{q}} \in \mathcal{S} \cap \mathcal{C}_{\nu}^{(0)}} & =\frac{\|\overline{\boldsymbol{q}}\|_{2}^{2}}{1-\|\overline{\boldsymbol{q}}\|_{2}^{2}} \\
\left.\mathcal{K}_{y}\right|_{\underline{\boldsymbol{q}} \in \mathcal{S} \cap \mathcal{C}_{\nu}^{(0)}} & =\left(1-\|\overline{\boldsymbol{q}}\|_{2}^{2}\right)^{2} \mathcal{K}_{s}^{(0)}+\sum_{k}\left\|\bar{q}^{(k)}\right\|_{4}^{4} \mathcal{K}_{s}^{(k)}
\end{aligned}
$$

where $\overline{\boldsymbol{q}}^{(k)}$ is used to denote the components of $\overline{\boldsymbol{q}}$ corresponding to user $k$.

In terms of the interference response, (14) becomes

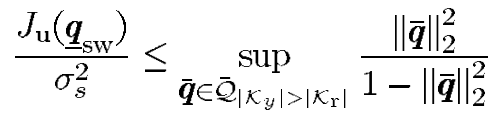

where $\overline{\mathcal{Q}}_{\left|\mathcal{K}_{y}\right|>\left|\mathcal{K}_{\mathrm{r}}\right|}$ is a translation of $\mathcal{Q}_{\left|\mathcal{K}_{3}\right|>\left|\mathcal{K}_{\mathrm{r}}\right|}$ into the interference space [implied by (13) and (15)]

$$
\begin{aligned}
\overline{\mathcal{Q}}_{\left|\mathcal{K}_{y}\right|>\left|\mathcal{K}_{\mathrm{r}}\right|}:= & \left\{\overline{\boldsymbol{q}} \in \overline{\mathcal{Q}}_{\text {CnS s.t. }} \mid\left(1-\|\bar{q}\|_{2}^{2}\right)^{2} \mathcal{K}_{s}^{(0)}\right. \\
& \left.+\sum_{k}|| \bar{q}^{(k)} \|_{4}^{4} \mathcal{K}_{s}^{(k)}|>| \mathcal{K}_{\mathrm{r}} \mid\right\}
\end{aligned}
$$

Finally, since $\|\overline{\boldsymbol{q}}\|_{2}^{2} /\left(1-\|\overline{\boldsymbol{q}}\|_{2}^{2}\right)$ is strictly increasing in $\|\overline{\boldsymbol{q}}\|_{2}$ (for $\overline{\boldsymbol{q}} \in \overline{\mathcal{Q}}_{\mathcal{C} \cap \mathcal{S}}$ ), (16) can be written as

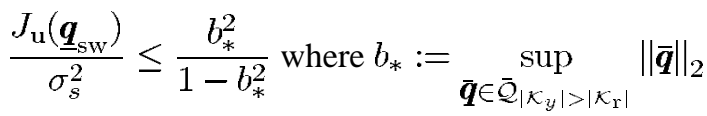

and thus, computation of the SW-UMSE bound reduces to computation of the quantity $b_{*}$.

Fig. 3(a) presents a summary of the bounding procedure in the interference response $(\overline{\boldsymbol{q}})$ space. The set of attainable interference responses is denoted by $\overline{\mathcal{Q}}_{\mathbf{a}}$, which can be interpreted as a projection of $\mathcal{Q}_{\mathrm{a}} \cap \mathcal{S} \cap \mathcal{C}_{\nu}^{(0)}$ onto the interference space. Notice that the reference response $\overline{\boldsymbol{q}}_{\mathrm{r}}$ and the SW response $\overline{\boldsymbol{q}}_{\mathrm{sW}}$ both lie in the attainable set $\overline{\mathcal{Q}}_{\mathrm{a}}$. Although the exact location of the kurtosis local maximum $\bar{q}_{\text {sw }}$ is unknown, we know that it lies within $\overline{\mathcal{Q}}_{\left|\mathcal{K}_{y}\right|>\left|\mathcal{K}_{\mathrm{r}}\right|}$, as shown in Fig. 3(a) by the interior of the shaded region. Thus, an upper bound on the UMSE of the SW estimator can be calculated using $b_{*}$, which is the supremal value of $\|\overline{\boldsymbol{q}}\|_{2}$ over $\overline{\boldsymbol{q}} \in \overline{\mathcal{Q}}_{\left|\mathcal{K}_{y}\right|>\left|\mathcal{K}_{\mathrm{r}}\right|}$.

Fig. 3(b) depicts the situation that may occur when the boundary of $\overline{\mathcal{Q}}_{\left|\mathcal{K}_{y}\right|>\left|\mathcal{K}_{\mathrm{r}}\right|}$ is not contained in $\overline{\mathcal{Q}}_{\mathcal{C} \cap \mathcal{S}}$. Essentially, there exists no attainable local $\left|\mathcal{K}_{y}\right|$ maximum inside $\overline{\mathcal{Q}}_{\mathrm{C}}$ S (i.e., for the desired user at delay $\nu$ ); $\left|\mathcal{K}_{y}\right|$ monotonically increases as we move northeast along $\overline{\mathcal{Q}}_{\mathrm{a}}$ toward the boundary of the open set $\overline{\mathcal{Q}}_{\mathcal{C}}$ S . Our existence conditions will be sufficient to avoid these cases.

\section{Statement of Bounds}

In this section, we present SW existence statements and SW-UMSE bounds deduced via the method described in Sections III-A and B. Proof details appear in the Appendix. We will use $\mathcal{K}_{\mathrm{m}}$ to denote the kurtosis of nor- 
(a)

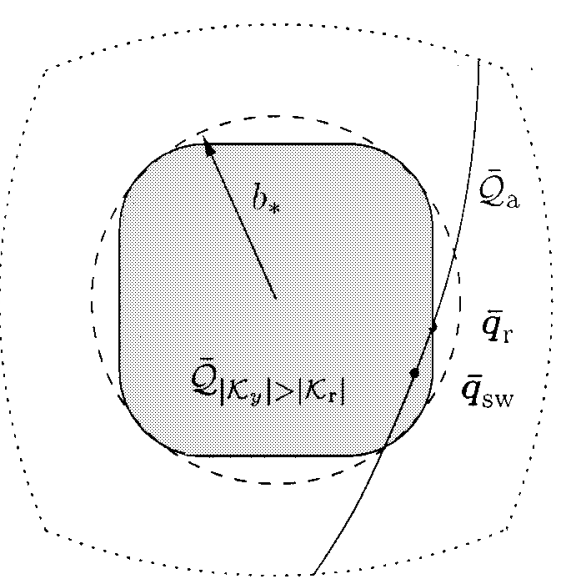

(b)

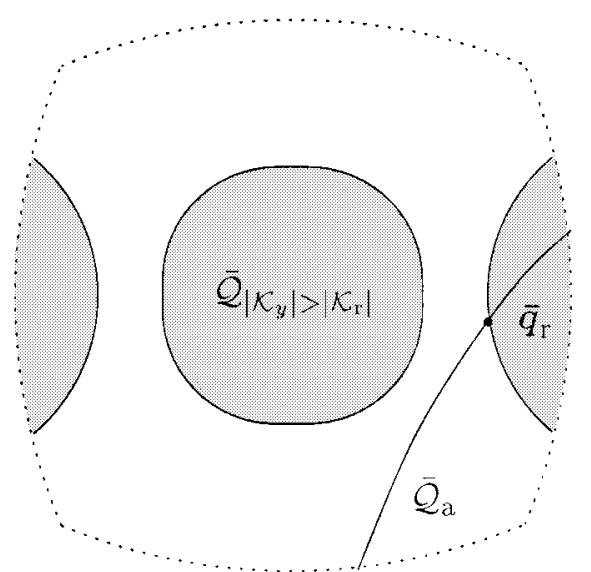

Fig. 3. Illustration of (a) SW-UMSE bounding technique in the $\overline{\boldsymbol{q}}$-space. (b) Case where no attainable local $\left|\mathcal{K}_{y}\right|$ maximum exists for the desired user/delay pair. Shaded regions denote $\overline{\mathcal{Q}}_{\left|\mathcal{K}_{y}\right|>\left|\mathcal{K}_{\mathbf{r}}\right|}$.

malized Wiener estimates, i.e., estimates associated with response $\underline{\boldsymbol{q}}_{\mathrm{r}}=\underline{\boldsymbol{q}}_{\mathrm{m}} /\left\|\underline{\boldsymbol{q}}_{\mathrm{m}}\right\|_{2}$, so that $\sigma_{y}^{2}=\sigma_{s}^{2}$. In addition, $\rho_{\mathrm{m}}:=\left|\mathcal{K}_{\mathrm{m}} / \mathcal{K}_{s}^{(0)}\right|, \rho_{\max }:=\max _{0 \leq k \leq K}\left|\mathcal{K}_{s}^{(k)} / \mathcal{K}_{s}^{(0)}\right|$, and $\rho_{\min }:=\min _{0 \leq k \leq K}\left(\mathcal{K}_{s}^{(k)} / \mathcal{K}_{s}^{(0)}\right)$.

Theorem 1: When the Wiener response lies in the dominant cone associated with the desired user at delay $\nu$ (i.e., $\underline{q}_{\mathrm{m}} \in \mathcal{C}_{\nu}^{(0)}$ ) and

$$
\left|\mathcal{K}_{s}^{(0)}\right| \geq\left|\mathcal{K}_{\mathrm{m}}\right|>\frac{\left(\left|\mathcal{K}_{s}^{(0)}\right|+\max _{k}\left|\mathcal{K}_{s}^{(k)}\right|\right)}{4}
$$

an SW estimator associated with the same user/delay exists, and its UMSE can be upper bounded by

$$
\left.J_{\mathrm{u}}\right|_{\mathrm{sw}} ^{\max , \mathcal{K}_{\mathrm{m}}}:=\frac{1-\sqrt{\left(\rho_{\max }+1\right) \rho_{\mathrm{m}}-\rho_{\max }}}{\rho_{\max }+\sqrt{\left(\rho_{\max }+1\right) \rho_{\mathrm{m}}-\rho_{\max }}} \sigma_{s}^{2} .
$$

As shown in the Appendix, the kurtosis requirement (19) requires sufficiently good Weiner performance.

While Theorem 1 presents a closed-form SW-UMSE bounding expression in terms of the kurtosis of MMSE estimates, it is also possible to derive lower and upper bounds in terms of the UMSE of the MMSE estimator. To do this, we rewrite (19) and (20) using a slightly weaker existence condition and looser bound. (See the Appendix for details of this procedure.)
Theorem 2: If $0 \leq J_{\mathbf{u}}\left(\underline{\boldsymbol{q}}_{\mathrm{m}}\right)<J_{\mathrm{o}} \sigma_{s}^{2}$, where

$$
J_{\mathrm{o}}:= \begin{cases}2 \sqrt{\left(1+\rho_{\max }\right)^{-1}-1,} & \rho_{\min } \geq 0 \\ \frac{3-\rho_{\max }}{\tilde{5}+\rho_{\max }}, & \rho_{\min }=-1 \\ \frac{1-\sqrt{1-\frac{\left(3-\rho_{\max }\right)\left(1+\rho_{\min }\right)}{4}}}{\rho_{\min }+\sqrt{1-\frac{\left(3-\rho_{\max }\right)\left(1+\rho_{\min }\right)}{4}}}, & \rho_{\min }<0, \rho_{\min } \neq-1\end{cases}
$$

then an SW estimator associated with the desired user at delay $\nu$ exists, and its UMSE can be bounded as

$$
J_{\mathbf{u}}\left(\underline{\boldsymbol{q}}_{\mathrm{m}}\right) \leq J_{\mathbf{u}}\left(\underline{\boldsymbol{q}}_{\mathrm{sw}}\right) \leq\left. J_{\mathbf{u}}\right|_{\mathrm{sw}} ^{\max , \mathcal{K}_{\mathrm{m}}} \leq\left. J_{\mathbf{u}}\right|_{\mathrm{sw}} ^{\max , J_{\mathrm{u}}\left(\underline{\underline{q}}_{\mathrm{m}}\right)} .
$$

where we have (22), shown at the bottom of the page.

Equation (22) leads to an elegant approximation of the extra UMSE of SW estimators

$$
\mathcal{E}_{\mathbf{u}}\left(\underline{\boldsymbol{q}}_{\mathrm{sw}}\right):=J_{\mathrm{u}}\left(\underline{\boldsymbol{q}}_{\mathrm{sw}}\right)-J_{\mathbf{u}}\left(\underline{\boldsymbol{q}}_{\mathrm{m}}\right) .
$$

Theorem 3: If $0 \leq J_{\mathbf{u}}\left(\underline{\boldsymbol{q}}_{\mathrm{m}}\right)<J_{\mathrm{o}} \sigma_{s}^{2}$, then the extra UMSE of SW estimators can be bounded as $\mathcal{E}_{\mathbf{u}}\left(\underline{\boldsymbol{q}}_{\mathrm{sw}}\right) \leq\left.\mathcal{E}_{\mathbf{u}}\right|_{\mathrm{c}} ^{\max , J_{\mathrm{u}}\left(\underline{\boldsymbol{q}}_{\mathrm{m}}\right)}$, where

$$
\begin{aligned}
& \left.\mathcal{E}_{\mathbf{u}}\right|_{\mathrm{c}} ^{\max , J_{\mathrm{u}}\left(\underline{\boldsymbol{q}}_{\mathrm{m}}\right)} \\
& :=\left.J_{\mathbf{u}}\right|_{\mathrm{sw}} ^{\max , J_{\mathrm{u}}\left(\underline{\boldsymbol{q}}_{\mathrm{m}}\right)}-J_{\mathbf{u}}\left(\underline{\boldsymbol{q}}_{\mathrm{m}}\right) \\
& = \begin{cases}\frac{1}{2 \sigma_{s}^{2}} \rho_{\max } J_{\mathbf{u}}^{2}\left(\underline{\boldsymbol{q}}_{\mathrm{m}}\right)+\mathcal{O}\left(J_{\mathbf{u}}^{3}\left(\underline{\boldsymbol{q}}_{\mathrm{m}}\right)\right), & \rho_{\min } \geq 0 \\
\frac{1}{2 \sigma_{s}^{2}}\left(\rho_{\max }-\rho_{\min }\right) J_{\mathbf{u}}^{2}\left(\underline{\boldsymbol{q}}_{\mathrm{m}}\right)+\mathcal{O}\left(J_{\mathbf{u}}^{3}\left(\underline{\boldsymbol{q}}_{\mathrm{m}}\right)\right), & \rho_{\min }<0 .\end{cases}
\end{aligned}
$$

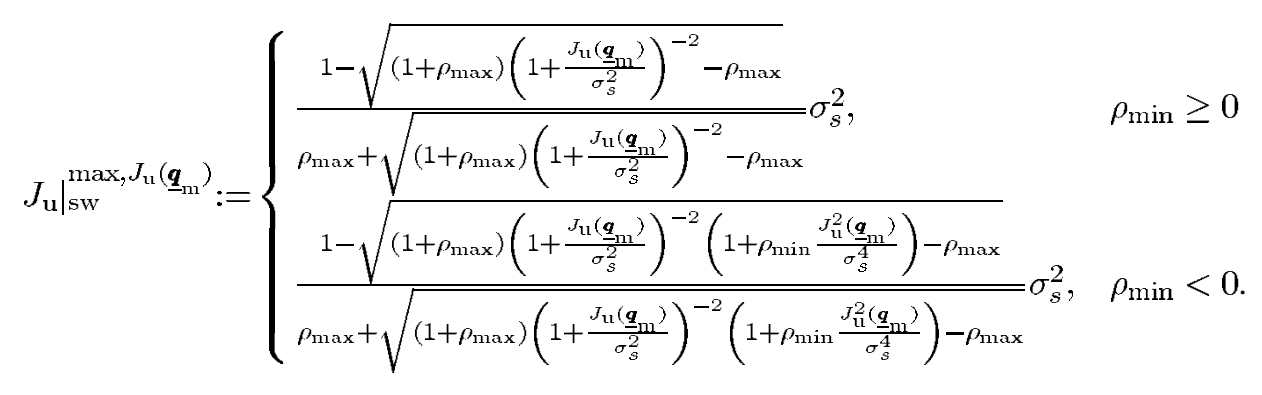



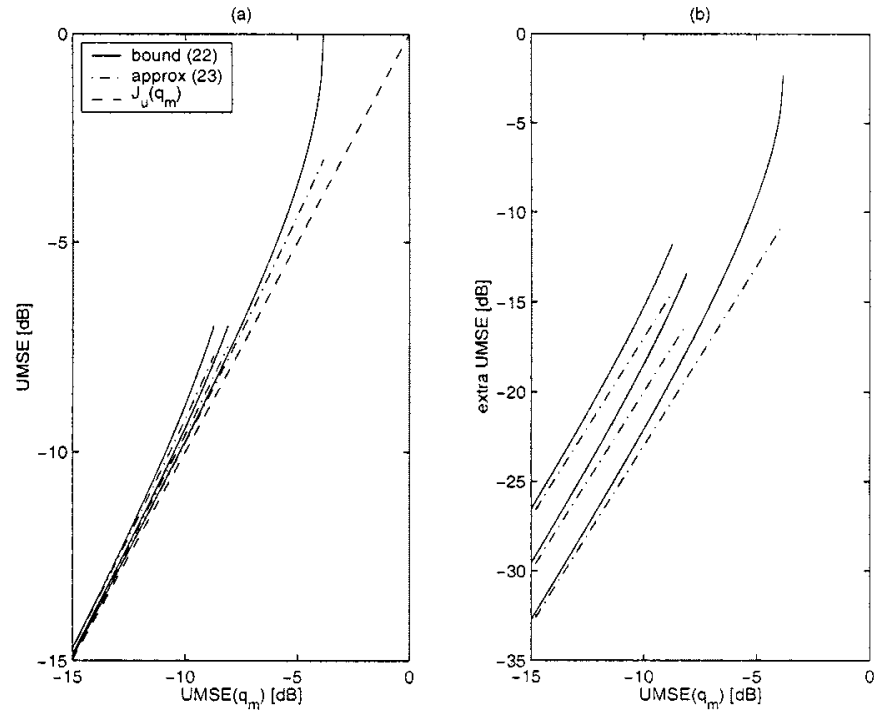

Fig. 4. Upper bound on (a) SW-UMSE and (b) extra SW-UMSE versus $J_{\mathbf{u}}\left(\underline{\boldsymbol{q}}_{\mathrm{m}}\right)$ (when $\sigma_{s}^{2}=1$ ) from (22) with second-order approximation from (23). From left to right, $\left\{\rho_{\min }, \rho_{\max }\right\}=\{-2,2\},\{0,2\}$, and $\{0,1\}$.

Equation (23) implies that the extra UMSE of SW estimators is upper bounded by approximately the square of the minimum UMSE. Fig. 4 plots the upper bound on SW-UMSE and extra SW-UMSE from (22) as a function of $J_{\mathbf{u}}\left(\underline{\boldsymbol{q}}_{\mathrm{m}}\right) / \sigma_{s}^{2}$ for various values of $\rho_{\min }$ and $\rho_{\max }$. The second-order approximation based on (23) appears very good for all but the largest values of UMSE.

\section{Comments on the SW-UMSE Bounds}

1) Implicit Incorporation of $\mathcal{Q}_{\mathrm{a}}$ : First, recall that the SW-UMSE bounding procedure incorporated $\mathcal{Q}_{\mathbf{a}}$, which is the set of attainable global responses, only through the requirement that $\underline{\boldsymbol{q}}_{\mathrm{r}} \in \mathcal{Q}_{\mathbf{a}}$. Thus, Theorems 1-3, which were written under the reference choice $\underline{\boldsymbol{q}}_{\mathrm{r}}=\underline{\boldsymbol{q}}_{\mathrm{m}} /\left\|\underline{\boldsymbol{q}}_{\mathrm{m}}\right\|_{2} \in \mathcal{Q}_{\mathrm{a}}$, implicitly incorporate the channel and/or estimator constraints that define $\mathcal{Q}_{\mathrm{a}}$. For example, if $\underline{\boldsymbol{q}}_{\mathrm{m}}$ is the MMSE response constrained to the set of length-two FIR estimators, then SW-UMSE bounds based on this $\underline{q}_{\mathrm{m}}$ will implicitly incorporate the length-two FIR constraint. The implicit incorporation of the attainable set $\mathcal{Q}_{\mathrm{a}}$ makes these bounding theorems quite general and easy to apply.

2) Effect of $\rho_{\min }$ and $\rho_{\max }$ : When $\rho_{\min } \geq 0$ and $\rho_{\max }=1$, the expressions in Theorems 1-3 simplify to

$$
\begin{aligned}
J_{\mathbf{u}}\left(\underline{\boldsymbol{q}}_{\mathrm{sW}}\right) & \leq \frac{1-\sqrt{2 \rho_{\mathrm{m}}-1}}{1+\sqrt{2 \rho_{\mathrm{m}}-1}} \sigma_{s}^{2} \text { for } \frac{1}{2}<\rho_{\mathrm{m}}<1 \\
& \leq \frac{1-\sqrt{2\left(1+\frac{J_{\mathrm{u}}\left(\underline{\boldsymbol{q}}_{\mathrm{m}}\right)}{\sigma_{s}^{2}}\right)^{-2}-1}}{1+\sqrt{2\left(1+\frac{J_{\mathrm{u}}\left(\underline{\boldsymbol{q}}_{\mathrm{m}}\right)}{\sigma_{s}^{2}}\right)^{-2}-1}} \sigma_{s}^{2} \\
\text { for } \frac{J_{\mathbf{u}}\left(\underline{\boldsymbol{q}}_{\mathrm{m}}\right)}{\sigma_{s}^{2}} & <\sqrt{2}-1 \\
& =J_{\mathbf{u}}\left(\underline{\boldsymbol{q}}_{\mathrm{m}}\right)+\frac{1}{2 \sigma_{s}^{2}} J_{\mathbf{u}}^{2}\left(\underline{\boldsymbol{q}}_{\mathrm{m}}\right)+\mathcal{O}\left(J_{\mathbf{u}}^{3}\left(\underline{\boldsymbol{q}}_{\mathrm{m}}\right)\right) .
\end{aligned}
$$

Note that the expressions above are independent of the specific distributions of the desired and interfering sources. The case $\rho_{\min } \geq 0$ occurs when either no sources are super-Gaussian or no sources are sub-Gaussian, whereas $\rho_{\max }=1$ occurs when there is no source with absolute kurtosis greater than that of the desired source.

In data communication models, it is typical that $\rho_{\min } \geq 0$ and $\rho_{\max }=1$. This corresponds, e.g., to the following common assumptions:

a) sub-Gaussian desired source in the presence of Gaussian noise;

b) constant-modulus desired source in the presence of nonsuper-Gaussian interference.

The case $\rho_{\max }>1$ might arise from a nonconstant-modulus desired source in the presence of constant modulus interference. Note that condition (19) is not able to guarantee the existence of a SW estimator for the desired user/delay when $\rho_{\max } \geq 3$.

3) Generalization of Perfect SW-Estimation Property: Finally, we note that the $J_{\mathbf{u}}\left(\underline{\boldsymbol{q}}_{\mathrm{m}}\right)$-based SW-UMSE bound in Theorem 2 implies that the perfect SW-estimation property, proven under more restrictive conditions in [1] extends to the general multisource linear model of Fig. 1.

Corollary 1: SW estimators are perfect (up to a scaling) when Wiener estimators are perfect.

Proof: From Theorem 2, $J_{\mathbf{u}}\left(\underline{q}_{\mathrm{m}}\right)=0 \Rightarrow J_{\mathbf{u}}\left(\underline{q}_{\mathrm{sw}}\right)=0$.

\section{NUMERICAL EXAMPLES}

Here, we present the results of experiments that compare the UMSE bounds of Theorems 1 and 2 with the UMSE characterizing SW estimators found by gradient descent ${ }^{3}$ under various source/interference environments. In all experiments, ten nonGaussian sources are linearly mixed using random (zero-mean Gaussian) mixture coefficients. The estimator observes the mixture at eight sensors in the presence of AWGN and generates linear estimates of a particular source using eight adjustable parameters. The AWGN is modeled using eight Gaussian sources (i.e., one per sensor) with channel gains chosen to yield a particular SNR. We define SNR as the ratio of total power received from the desired source to the AWGN power at each sensor. Note that with eight sensors and 18 sources, $\mathcal{H}$ is not full column rank, and perfect estimation is not possible.

Figs. 5(a)-8(a) plot the UMSE upper bounds $\left.J_{\mathbf{u}}\right|_{\mathrm{sw}} ^{\max }, \mathcal{K}_{\mathrm{m}}$ and $\left.J_{\mathbf{u}}\right|_{\mathrm{sw}} ^{\max , J_{\mathrm{u}}\left(\underline{\boldsymbol{q}}_{\mathrm{m}}\right)}$ for comparison with $J_{\mathbf{u}}\left(\underline{\boldsymbol{q}}_{\mathrm{sw}}\right)$. As a means of "zooming in" on the small differences in UMSE, Figs. 5(b)-8(b) plot the extra-UMSE upper bounds $\left.\mathcal{E}_{\mathbf{u}}\right|_{\mathrm{c}} ^{\max , \mathcal{K}_{\mathrm{m}}}$ and $\left.\mathcal{E}_{\mathrm{u}}\right|_{\mathrm{c}} ^{\max , J_{\mathrm{u}}\left(\underline{\boldsymbol{q}}_{\mathrm{m}}\right)}$. In all plots, the $J_{\mathrm{u}}\left(\underline{\boldsymbol{q}}_{\mathrm{m}}\right)$-based bounds are denoted by solid lines, the $\mathcal{K}_{\mathrm{m}}$-based bounds are denoted by $\bullet$, and the gradient-descent values are denoted by $\times$.

In Fig. 5, ten BPSK sources (i.e., $\mathcal{K}_{s}^{(k)}=-2$ ) mix with Gaussian noise, corresponding to $\rho_{\min }=0$ and $\rho_{\max }=1$. Note, from Fig. 5(a), the tightness of the bounds for all but the largest values of $J_{\mathbf{u}}\left(\underline{\boldsymbol{q}}_{\mathrm{m}}\right)$. Fig. 6 considers ten super-Gaussian sources, with $\mathcal{K}_{s}^{(k)}=2$, in the presence of Gaussian noise. From (22), we do not expect SW performance to differ from the previous experiment since $\rho_{\min }$ and $\rho_{\max }$ stay the same. This notion is confirmed by comparison of Figs. 5 and 6 .

${ }^{3}$ Gradient descent results were obtained by the MATLAB routine "fmincon," which was initialized randomly in a small ball around the MMSE estimator. 

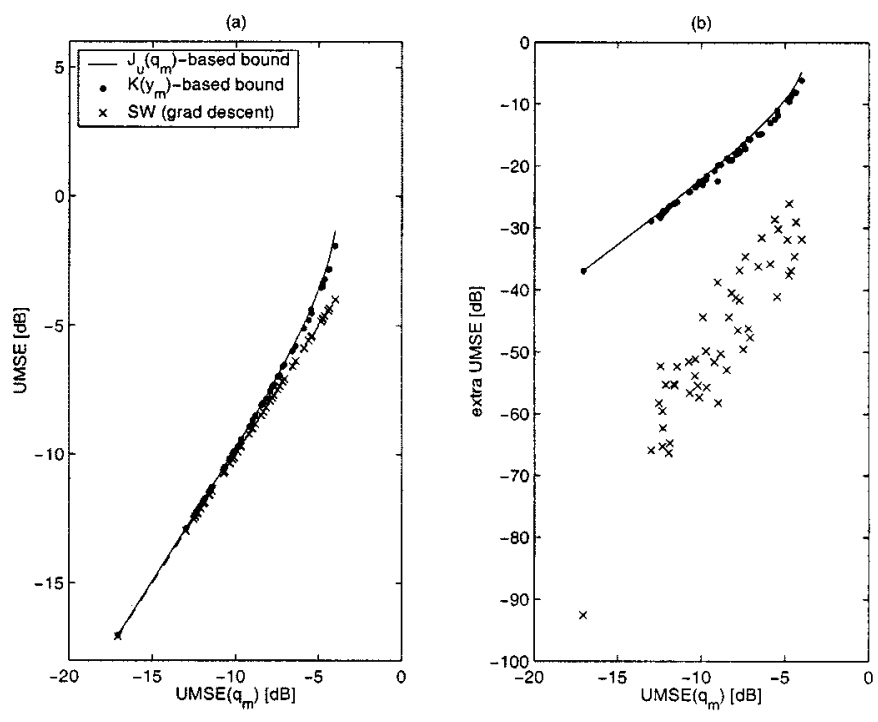

Fig. 5. Bounds on SW-UMSE for eight sensors, ten randomly mixed BPSK sources, and AWGN at $-20 \mathrm{~dB}$.
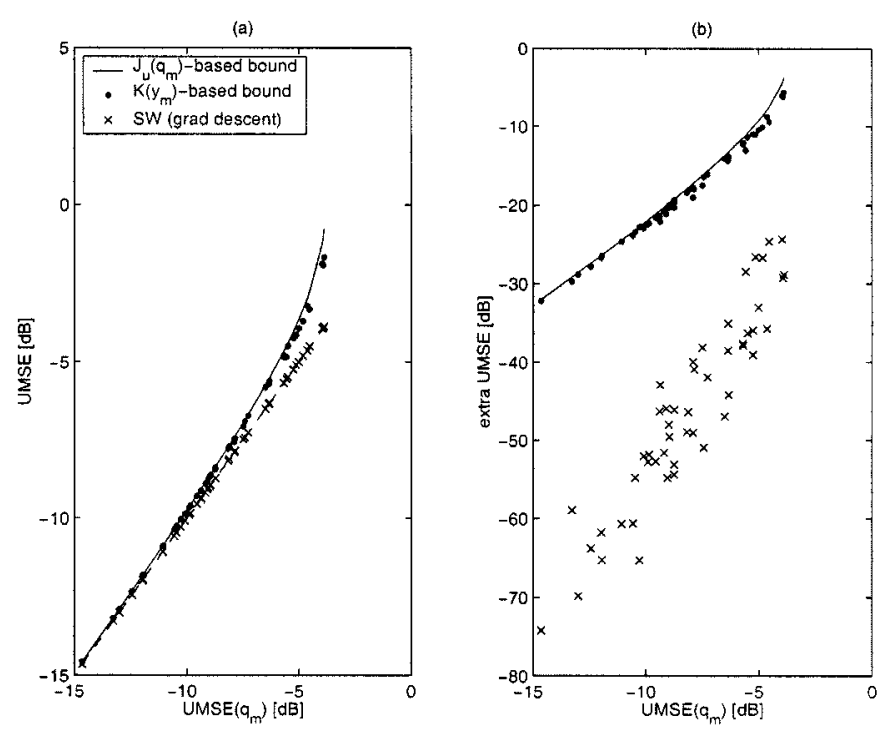

Fig. 6. Bounds on SW-UMSE for eight sensors, ten randomly mixed sources with $\mathcal{K}_{s}^{(k)}=2$, and AWGN at $-20 \mathrm{~dB}$.

Fig. 7 examines the estimation of a near-Gaussian signal $\left(\mathcal{K}_{s}^{(0)}=0.8\right)$ in the presence of BPSK and AWGN interference, corresponding to $\rho_{\min }=-2.5$ and $\rho_{\max }=2.5$. Comparing this experiment with the previous two, notice that here, $\left.J_{\mathbf{u}}\right|_{\mathrm{sw}} ^{\max }, \mathcal{K}_{\mathrm{m}}$ is appreciably tighter than $\left.J_{\mathbf{u}}\right|_{\mathrm{sw}} ^{\max , J_{\mathrm{u}}\left(\underline{\boldsymbol{q}}_{\mathrm{n}}\right)}$ for larger values of $J_{\mathbf{u}}\left(\underline{q}_{\mathrm{m}}\right)$. Finally, Fig. 8 examines the performance of a super-Gaussian signal $\left(\mathcal{K}_{s}^{(0)}=3\right)$ in the presence of impulsive-type noise $\left(\mathcal{K}_{s}^{(k)}=8\right)$. When $\rho_{\max } \gg 1$, (19) and (21) imply that we can only guarantee the existence of SW estimators in situations where MMSE estimators are relatively good. As the interference environment in this experiment corresponds to $\rho_{\max }=2.67$, UMSE bounds exist only when $J_{\mathbf{u}}\left(\underline{\boldsymbol{q}}_{\mathrm{m}}\right)$ is less than about $-13 \mathrm{~dB}$.
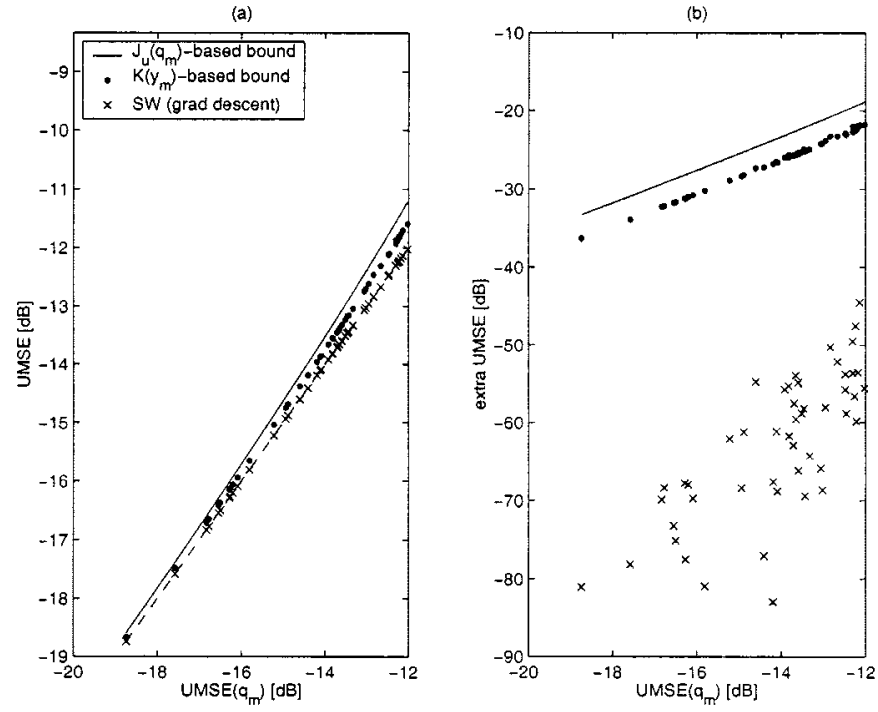

Fig. 7. Bounds on SW-UMSE for eight sensors, five randomly mixed BPSK sources, and five randomly mixed sources with $\mathcal{K}_{s}^{(k)}=0.8$ (one of which is desired), and AWGN at $-20 \mathrm{~dB}$.
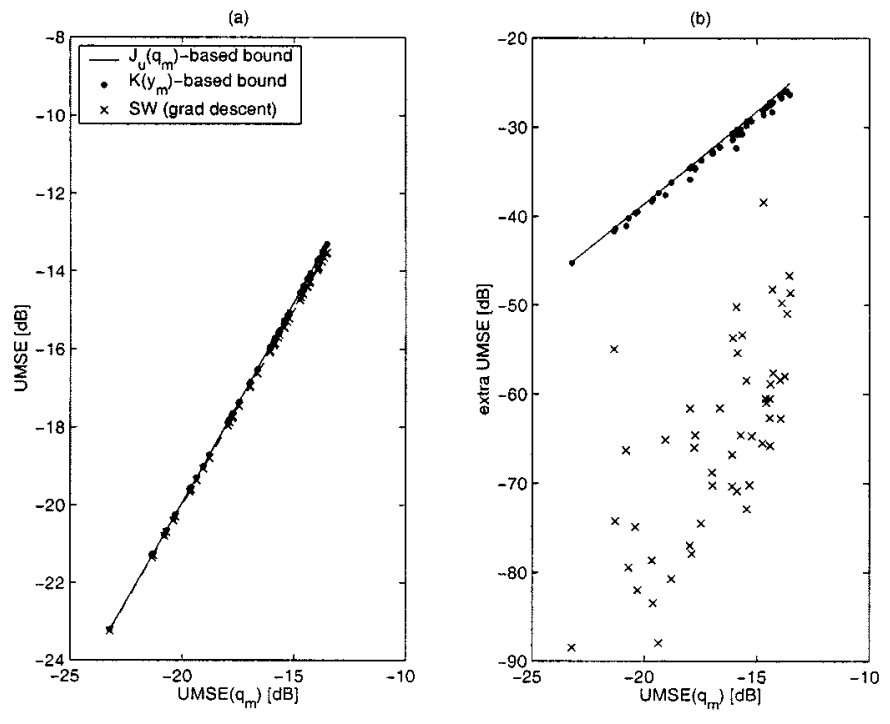

Fig. 8. Bounds on SW-UMSE for eight sensors, five randomly mixed sources with $\mathcal{K}_{s}^{(k)}=8$, five randomly mixed sources with $\mathcal{K}_{s}^{(k)}=3$ (one of which is desired), and AWGN at $-30 \mathrm{~dB}$.

\section{CONCLUSION}

In this paper, we have derived sufficient conditions under which SW estimators exist and derived upper bounds for the UMSE of SW estimators. The existence conditions are simple tests that guarantee an SW estimator for the desired user at a particular delay. All results have been proven for vector-valued IIR channels and constrained vector-valued FIR estimators. The first existence/bound pair is a function of the kurtosis of the MMSE estimates, whereas the second existence/bound pair is a function of the minimum (i.e., Wiener) UMSE. Analysis of the second bound shows that the extra UMSE of SW estimators is upper bounded by approximately the square of the minimum UMSE. Thus, SW estimators are very close (in a MSE sense) to optimum linear estimators when the minimum MSE is small. 
Numerical simulations suggest that the bounds are reasonably tight.

\section{APPENDIX}

\section{DERIVATION DETAILS FOR SW-UMSE BOUNDS}

\section{A. Proof of Theorem 1}

To establish the existence of $\overline{\boldsymbol{q}}_{\mathrm{sw}}$, which is an attainable local $\left|\mathcal{K}_{y}\right|$ maximum within $\overline{\mathcal{Q}}_{\mathcal{C} \cap \mathcal{S}}$, we appeal to the Weierstrass theorem [10, p. 40], which states that a continuous cost functional must have a local maximum on a compact set if there exist points in the interior that give cost strictly higher than anywhere on the boundary. If we can show that the "interior" set $\overline{\mathcal{Q}}_{\left|\mathcal{K}_{y}\right|>\left|\mathcal{K}_{\mathrm{r}}\right|} \cap \overline{\mathcal{Q}}_{\mathrm{a}}$ exists and that its closure forms a compact set within $\mathcal{Q}_{\mathcal{C} \cap \mathcal{S}}$, then the continuous cost functional $\left|\mathcal{K}_{y}(\overline{\boldsymbol{q}})\right|$ and the boundary cost $\left|\mathcal{K}_{\mathrm{r}}\right|$ suffice to prove the existence of $\overline{\boldsymbol{q}}_{\mathrm{sw}}$, which is an attainable local maximum of $\left|\mathcal{K}_{y}\right|$ within $\overline{\mathcal{Q}}_{\mathcal{C} \cap \mathcal{S}}$. Our existence proof will consist of three steps: deriving conditions under which

i) closure $\left(\overline{\mathcal{Q}}_{\left|\mathcal{K}_{y}\right|>\left|\mathcal{K}_{\mathrm{r}}\right|} \cap \overline{\mathcal{Q}}_{\mathrm{a}}\right) \subset \overline{\mathcal{Q}}_{\mathcal{C} \mathcal{S}}$;

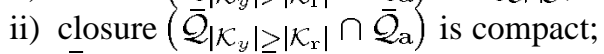

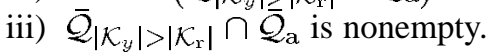

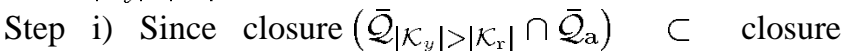
$\left(\overline{\mathcal{Q}}_{\left|\mathcal{K}_{y}\right|>\left|\mathcal{K}_{\mathrm{r}}\right|}\right)$, condition i) is implied by the following equivalent statements. [Regarding the equivalence, recall (17) and the fact that $\overline{\mathcal{Q}}_{\mathcal{C} N \mathcal{S}}$ is open].

$$
\begin{aligned}
& \operatorname{closure}\left(\overline{\mathcal{Q}}_{\left|\mathcal{K}_{y}\right|>\left|\mathcal{K}_{\mathrm{r}}\right|} \mid \subset \overline{\mathcal{Q}}_{\mathcal{C} \cap \mathcal{S}}\right. \\
& \quad \Leftrightarrow\left\{\bar{q} \in \operatorname{closure}\left(\overline{\mathcal{Q}}_{\mathcal{C}}\right):\left|\mathcal{K}_{y}(\overline{\boldsymbol{q}})\right| \geq\left|\mathcal{K}_{\mathrm{r}}\right|\right\} \subset \overline{\mathcal{Q}}_{\mathcal{C} \cap \mathcal{S}} \\
& \quad \Leftrightarrow \max _{\overline{\boldsymbol{q}} \in \operatorname{bndr}\left(\overline{\mathcal{Q}}_{\mathcal{C} \cap \mathcal{S}}\right)}\left|\mathcal{K}_{y}(\overline{\boldsymbol{q}})\right|<\left|\mathcal{K}_{\mathrm{r}}\right| .
\end{aligned}
$$

We focus now on (24). By definition, points $\overline{\boldsymbol{q}}$ on the boundary of $\overline{\mathcal{Q}}_{\mathcal{C} \cap \mathcal{S}}$ correspond to $q$ such that $\left|q_{\nu}^{(0)}\right|=\max _{(\ell, \delta) \neq(0, \nu)}\left|q_{\delta}^{(\ell)}\right|$ and $\|\underline{q}\|_{2}^{2}=1$. By evaluating gradient and Hessian, it can be shown ${ }^{4}$ that

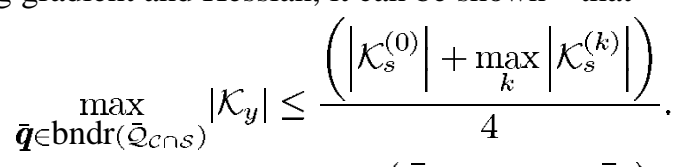

Thus, we can guarantee closure $\left(\overline{\mathcal{Q}}_{\left|\mathcal{K}_{y}\right|>\left|\mathcal{K}_{\mathbf{r}}\right|} \cap \overline{\mathcal{Q}}_{\mathbf{a}}\right) \subset \overline{\mathcal{Q}}_{\mathrm{C} \cap \mathcal{S}}$ when

$$
\left|\mathcal{K}_{\mathrm{r}}\right|>\frac{\left(\left|\mathcal{K}_{s}^{(0)}\right|+\max _{k}\left|\mathcal{K}_{s}^{(k) \mid}\right|\right)}{4} .
$$

Step ii) closure $\left(\overline{\mathcal{Q}}_{\left|\mathcal{K}_{y}\right|>\left|\mathcal{K}_{\mathrm{r}}\right|} \cap \overline{\mathcal{Q}}_{\mathrm{a}}\right)$ is compact under (25) since it is contained in the bounded set $\overline{\mathcal{Q}}_{\mathcal{C} \cap \mathcal{S}}$ and since $\bar{q}$ lies in a finite dimensional subspace of $\ell_{2}$.

Step iii) We now show that the presence of an attainable reference in the desired cone and on the sphere (i.e., $\underline{\boldsymbol{q}}_{\mathrm{r}} \in$ $\mathcal{Q}_{\mathrm{a}} \cap \mathcal{C}_{\nu}^{(0)} \cap \mathcal{S}$ ) coupled with the condition

$$
\left|\mathcal{K}_{s}^{(0)}\right|>\left|\mathcal{K}_{\mathrm{r}}\right|>\frac{1}{4}\left(\left|\mathcal{K}_{s}^{(0)}\right|+\max _{k}\left|\mathcal{K}_{s}^{(k)}\right|\right)
$$

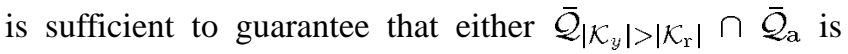
nonempty or that $\underline{q}_{\mathrm{r}}=\underline{\boldsymbol{q}}_{\mathrm{sw}}$. When the right inequality in

${ }^{4}$ Contact the corresponding author for details of all gradient/Hessian analyzes mentioned in this appendix.
(26) is satisfied, gradient and Hessian arguments can be used to show that $\overline{\boldsymbol{q}}=0$ is the unique global maximizer of $\left|\mathcal{K}_{y}\right|$ over $\overline{\mathcal{Q}}_{\mathcal{C}}$ S , yielding a maximum value of $\left|\mathcal{K}_{s}^{(0)}\right|$. Thus, when the left inequality in (26) is satisfied, there exists a set of $\overline{\boldsymbol{q}} \in \overline{\mathcal{Q}}_{\mathcal{C} \cap \mathcal{S}}$ for which $\left|\mathcal{K}_{y}(\overline{\boldsymbol{q}})\right|>\left|\mathcal{K}_{\mathrm{r}}\right|$, implying [via definition (17)] that $\overline{\mathcal{Q}}_{\left|\mathcal{K}_{y}\right|>\left|\mathcal{K}_{\mathrm{r}}\right|}$ is nonempty. If we can now show that $\overline{\mathcal{Q}}_{\mathrm{a}}$ intersects the interior of (open) $\overline{\mathcal{Q}}_{\left|\mathcal{K}_{y}\right|>\left|\mathcal{K}_{\mathrm{r}}\right|}$,

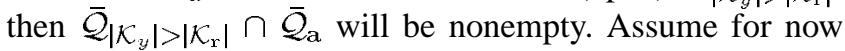
that $\overline{\boldsymbol{q}}_{\mathbf{r}} \neq \overline{\boldsymbol{q}}_{\text {sw }}$, i.e., $\overline{\boldsymbol{q}}_{\mathrm{r}}$ is not a local $\left|\mathcal{K}_{y}\right|$ maximum over $\overline{\mathcal{Q}}_{\mathrm{a}} \cap \overline{\mathcal{Q}}_{\mathcal{C} \cap \mathcal{S}}$. (We will treat the other case momentarily.) Then, there must exist a neighborhood of $\overline{\mathcal{Q}}_{\mathrm{a}} \cap \overline{\mathcal{Q}}_{\mathcal{C} \cap \mathcal{S}}$ containing $\overline{\boldsymbol{q}}_{\mathrm{r}}$ over which $\left|\mathcal{K}_{y}(\overline{\boldsymbol{q}})\right|>\left|\mathcal{K}_{\mathrm{r}}\right|$. The previous expression implies that $\overline{\mathcal{Q}}_{\mathrm{a}} \cap \overline{\mathcal{Q}}_{\left|\mathcal{K}_{y}\right|>\left|\mathcal{K}_{\mathrm{r}}\right|}$ is nonempty. Note that if the left inequality in (26) was replaced by an equality (meaning that $\underline{\boldsymbol{q}}_{\mathrm{r}}$ provides perfect blind estimation), $\overline{\mathcal{Q}}_{\left|\mathcal{K}_{y}\right|>\left|\mathcal{K}_{\mathrm{r}}\right|}$ becomes empty, and the previous arguments do not apply. However, as this $\overline{\boldsymbol{q}}_{\mathrm{r}}\left(\in \overline{\mathcal{Q}}_{\mathrm{a}}\right)$ is the unique global $\left|\mathcal{K}_{y}\right|$ maximizer over $\overline{\mathcal{Q}}_{\mathcal{C} N \mathcal{S}}$, it is clear that $\overline{\boldsymbol{q}}_{\mathrm{sw}}=\overline{\boldsymbol{q}}_{\mathrm{r}}$, giving SW existence and zero SW-UMSE [consistent with bound (20) for $\rho_{\mathrm{m}}=1$ ].

To conclude, (26) and the condition $\overline{\boldsymbol{q}}_{\mathrm{r}} \neq \overline{\boldsymbol{q}}_{\mathrm{sw}}$ suffice for our three-step approach to the application of the Weierstrass theorem, implying the existence of $\underline{q}_{\mathrm{sw}} \in \mathcal{Q}_{\mathrm{a}} \cap \mathcal{S} \cap \mathcal{C}_{\nu}^{(0)}$. If, on the other hand, $\overline{\boldsymbol{q}}_{\mathrm{r}}=\overline{\boldsymbol{q}}_{\mathrm{sw}}$, then the requirement that $\overline{\boldsymbol{q}}_{\mathrm{r}} \in \overline{\mathcal{Q}}_{\mathrm{a}} \cap \overline{\mathcal{Q}}_{\mathcal{C} \cap \mathcal{S}}$ implies the existence of $\underline{\boldsymbol{q}}_{\mathrm{sw}} \in \mathcal{Q}_{\mathrm{a}} \cap \mathcal{S} \cap \mathcal{C}_{\nu}^{(0)}$ directly.

The SW-UMSE upper bound in Theorem 1 follows from upper bounding the maximum interference radius $b_{*}$ defined in (18). For $\overline{\boldsymbol{q}} \in \overline{\mathcal{Q}}_{\left|\mathcal{K}_{y}\right|>\left|\mathcal{K}_{\mathrm{r}}\right|}$, (17) and the property $\|\overline{\boldsymbol{q}}\|_{4}^{4} \leq\|\overline{\boldsymbol{q}}\|_{2}^{4}$ guarantee

$$
\begin{aligned}
\left|\mathcal{K}_{\mathrm{r}}\right| & <\left|\left(1-\|\left.\overline{\boldsymbol{q}}\right|_{2} ^{2}\right)^{2} \mathcal{K}_{s}^{(0)}+\sum_{k}\left\|\overline{\boldsymbol{q}}^{(k)}\right\|_{4}^{4} \mathcal{K}_{s}^{(k)}\right| \\
& \leq\left(1-\|\overline{\boldsymbol{q}}\|_{2}^{2}\right)^{2}\left|\mathcal{K}_{s}^{(0)}\right|+\|\left.\overline{\boldsymbol{q}}\right|_{2} ^{4} \max _{k}\left|\mathcal{K}_{s}^{(k)}\right| \\
\Leftrightarrow 0 & <\|\overline{\boldsymbol{q}}\|_{2}^{4}\left(1+\rho_{\max }\right)-2 \|\left.\overline{\boldsymbol{q}}\right|_{2} ^{2}+\left(1-\rho_{\mathrm{r}}\right)
\end{aligned}
$$

where we arrive at (28) by dividing the previous inequality by $\left|\mathcal{K}_{s}^{(0)}\right|$ and substituting $\rho_{\mathrm{r}}=\left|\mathcal{K}_{\mathrm{r}}\right| /\left|\mathcal{K}_{s}^{(0)}\right|$ and $\rho_{\max }=$ $\max _{k}\left|\mathcal{K}_{s}^{(k)}\right| /\left|\mathcal{K}_{s}^{(0)}\right|$. Since the roots of $x^{2}\left(1+\rho_{\max }\right)-2 x+$ $\left(1-\rho_{\mathrm{r}}\right)$ are given by

$$
\left\{x_{1}, x_{2}\right\}=\frac{1 \pm \sqrt{\rho_{\mathrm{r}}\left(1+\rho_{\max }\right)-\rho_{\max }}}{1+\rho_{\max }}
$$

the $\|\overline{\boldsymbol{q}}\|_{2}^{2}$ satisfying (28) lie outside the closed interval $\left[x_{1}, x_{2}\right]$. Thus, $\overline{\mathcal{Q}}_{\left|\mathcal{K}_{y}\right|>\left|\mathcal{K}_{\mathrm{r}}\right|}$ is contained by $\left\{\overline{\boldsymbol{q}} \in \overline{\mathcal{Q}}_{\mathrm{C} \cap \mathcal{S}}:\|\overline{\boldsymbol{q}}\|_{2}^{2}<x_{1}\right\} \cup$ $\left\{\overline{\boldsymbol{q}} \in \overline{\mathcal{Q}}_{\mathrm{C} \cap \mathcal{S}}:\|\overline{\boldsymbol{q}}\|_{2}^{2}>x_{2}\right\}$.

We will now show that there is no component of $\overline{\mathcal{Q}}_{\left|\mathcal{K}_{y}\right|>\left|\mathcal{K}_{\mathrm{r}}\right|}$ that intersects $\left\{\overline{\boldsymbol{q}} \in \overline{\mathcal{Q}}_{\mathrm{C} \cap \mathcal{S}}:\|\overline{\boldsymbol{q}}\|_{2}^{2}>x_{2}\right\}$, implying that an upper bound for $b_{*}^{2}$, which is the largest $\|\left.\overline{\boldsymbol{q}}\right|_{2} ^{2}$ in $\overline{\mathcal{Q}}_{\left|\mathcal{K}_{y}\right|>\left|\mathcal{K}_{\mathrm{r}}\right|}$, is given by $x_{1}$. First, it is straightforward to verify that the existence condition (26) guarantees $0<x_{1}<0.5$ and $x_{2}>0.5$. Analyzing gradient and Hessian, it can be shown that the supremum of $\left|\mathcal{K}_{y}\right|$ over the (open) set $\left\{\overline{\boldsymbol{q}} \in \overline{\mathcal{Q}}_{\mathcal{C} \cap S}:\|\overline{\boldsymbol{q}}\|_{2}^{2}>0.5\right\}$ attains the 
value $\left(\left|\mathcal{K}_{s}^{(0)}\right|+\max _{k}\left|\mathcal{K}_{s}^{(k)}\right|\right) / 4$. Then, from existence condition (26) and the definition (17), we know that $\overline{\mathcal{Q}}_{\left|\mathcal{K}_{y}\right|>\left|\mathcal{K}_{\mathrm{r}}\right|}$ will not intersect $\left\{\overline{\boldsymbol{q}} \in \overline{\mathcal{Q}}_{\mathcal{C} \cap \mathcal{S}}:\|\bar{q}\|_{2}^{2}>0.5\right\}$.

The fact that $b_{*}$ is upper bounded by the smaller root $x_{1}$ implies the following bound:

$$
\begin{aligned}
\frac{J_{\mathbf{u}}\left(\underline{\boldsymbol{q}}_{\mathrm{sw}}\right)}{\sigma_{s}^{2}} & \leq \frac{b_{*}^{2}}{1-b_{*}^{2}} \\
& \leq \frac{x_{1}^{2}}{1-x_{1}^{2}}=\frac{1-\sqrt{\rho_{\mathrm{r}}\left(1+\rho_{\max }\right)-\rho_{\max }}}{\rho_{\max }+\sqrt{\rho_{\mathrm{r}}\left(1+\rho_{\max }\right)-\rho_{\max }}}
\end{aligned}
$$

Choosing the normalized Weiner reference $\underline{q}_{\mathrm{r}}=\underline{q}_{\mathrm{m}} /\left\|\underline{q}_{\mathrm{m}}\right\|_{2} \in$ $\mathcal{Q}_{\mathrm{a}} \cap \mathcal{S} \cap \mathcal{C}_{\nu}^{(0)}$ in (29) leads to Theorem 1. Note that this choice requires that the Weiner response lies in the dominant cone of the desired user/delay.

\section{B. Proof of Theorem 2}

From the Appendix, we know that the expressions in Theorem 1 hold for any estimates generated by a reference response $\underline{q}_{\mathrm{r}} \in \mathcal{Q}_{\mathrm{a}} \cap \mathcal{S} \cap \mathcal{C}_{\nu}^{(0)}$ generating kurtosis $\mathcal{K}_{\mathrm{r}}$. For such $\underline{\boldsymbol{q}}_{\mathrm{r}}$, the kurtosis-based UMSE upper bound appears in (29) and the existence condition in (26). [The Appendix separately established SW existence and perfect blind estimation when $\left|\mathcal{K}_{s}^{(0)}\right|=\left|\mathcal{K}_{\mathrm{r}}\right|$, corresponding to the case $J_{\mathbf{u}}\left(\underline{\boldsymbol{q}}_{\mathrm{m}}\right)=J_{\mathbf{u}}\left(\underline{\boldsymbol{q}}_{\mathrm{sw}}\right)=0$. For the remainder of this proof, however, we focus on the case $\left|\mathcal{K}_{s}^{(0)}\right|>$ $\left|\mathcal{K}_{\mathrm{r}}\right|$ as represented by (26).] Noting that $\left.J_{\mathbf{u}}\right|_{\mathrm{sw}} ^{\max }, \mathcal{K}_{\mathrm{r}}$ is a strictly decreasing function of $\rho_{\mathrm{r}}$ (over its valid range), an upper bound for $\left.J_{\mathbf{u}}\right|_{\mathrm{sw}} ^{\max , \mathcal{K}_{\mathrm{r}}}$ follows from a lower bound of $\rho_{\mathrm{r}}$. From (15)

$$
\begin{aligned}
\frac{\mathcal{K}_{\mathrm{r}}}{\mathcal{K}_{s}^{(0)}} & =\left(1-\left\|\overline{\boldsymbol{q}}_{\mathrm{r}}\right\|_{2}^{2}\right)^{2}+\sum_{k}\left\|\overline{\boldsymbol{q}}_{\mathrm{r}}^{(k)}\right\|_{4}^{4} \frac{\mathcal{K}_{s}^{(k)}}{\mathcal{K}_{s}^{(0)}} \\
& \geq\left(1-\left\|\overline{\boldsymbol{q}}_{\mathrm{r}}\right\|_{2}^{2}\right)^{2}+\min _{k}\left(\frac{\mathcal{K}_{s}^{(k)}}{\mathcal{K}_{s}^{(0)}}\right)\left\|\overline{\boldsymbol{q}}_{\mathrm{r}}\right\|_{4}^{4}
\end{aligned}
$$

Using $\rho_{\min }=\min _{k}\left(\mathcal{K}_{s}^{(k)} / \mathcal{K}_{s}^{(0)}\right),\|\bar{q}\|_{4}^{4} \leq\|\overline{\boldsymbol{q}}\|_{2}^{4}$, and $\rho_{\mathrm{r}}>$ $\left(1+\rho_{\max }\right) / 4$ from (26), we have the equation at the bottom of the page.

When $\rho_{\min } \geq 0$, we see that

$$
\begin{aligned}
1-2\left\|\overline{\boldsymbol{q}}_{\mathrm{r}}\right\|_{2}^{2}+\left\|\overline{\boldsymbol{q}}_{\mathrm{r}}\right\|_{2}^{4} & =\left(1-\left\|\overline{\boldsymbol{q}}_{\mathrm{r}}\right\|_{2}^{2}\right)^{2}=\left(1+\frac{\left\|\overline{\boldsymbol{q}}_{\mathrm{r}}\right\|_{2}^{2}}{1-\left\|\overline{\boldsymbol{q}}_{\mathrm{r}}\right\|_{2}^{2}}\right)^{-2} \\
& =\left(1+\frac{J_{\mathbf{u}}\left(\underline{\boldsymbol{q}}_{\mathrm{r}}\right)}{\sigma_{s}^{2}}\right)^{-2}
\end{aligned}
$$

which implies the bound

$$
\begin{aligned}
& \frac{\left.J_{\mathbf{u}}\right|_{\mathrm{SW}} ^{\max }, \mathcal{K}_{\mathrm{r}}}{\sigma_{s}^{2}} \\
& \leq \frac{1-\sqrt{\left(1+\rho_{\max }\right)\left(1+\frac{J_{\mathrm{u}}\left(\underline{\underline{q}}_{\mathrm{r}}\right)}{\sigma_{s}^{2}}\right)^{-2}-\rho_{\max }}}{\rho_{\max }+\sqrt{\left(1+\rho_{\max }\right)\left(1+\frac{J_{\mathrm{u}}\left(\underline{\boldsymbol{q}}_{\mathrm{r}}\right)}{\sigma_{s}^{2}}\right)^{-2}-\rho_{\max }}} .
\end{aligned}
$$

The existence condition (26) can also be rewritten using MMSEbased quantities. We first rewrite (26) in terms of $\rho_{\mathrm{r}}$ and $\rho_{\max }$.

$$
1>\rho_{\mathrm{r}}>\frac{1+\rho_{\max }}{4}
$$

Having just shown that $\rho_{\mathrm{r}} \geq\left(1+J_{\mathbf{u}}\left(\underline{\boldsymbol{q}}_{\mathrm{r}}\right) / \sigma_{s}^{2}\right)^{-2}$ when $\rho_{\min } \geq 0$, a sufficient condition for the right inequality of (31) is $\left(1+J_{\mathbf{u}}\left(\underline{q}_{\mathbf{r}}\right) / \sigma_{s}^{2}\right)^{-2}>\left(1+\rho_{\max }\right) / 4$, which can be restated as

$$
\frac{J_{\mathbf{u}}\left(\underline{\boldsymbol{q}}_{\mathbf{r}}\right)}{\sigma_{s}^{2}}<-1+2 \sqrt{\left(1+\rho_{\max }\right)^{-1}}
$$

For the left inequality in (31), we divide (27) by $\left|\mathcal{K}_{s}^{(0)}\right|$ to see that

$$
\begin{aligned}
\rho_{\mathrm{r}} & <1-2\left\|\overline{\boldsymbol{q}}_{\mathrm{r}}\right\|_{2}^{2}+\left(1+\rho_{\max }\right)\left\|\overline{\boldsymbol{q}}_{\mathrm{r}}\right\|_{2}^{4} \\
& =\left(1-\left\|\overline{\boldsymbol{q}}_{\mathrm{r}}\right\|_{2}^{2}\right)^{2}\left(1+\rho_{\max } \frac{\left\|\underline{\boldsymbol{q}}_{\mathrm{r}}\right\|_{2}^{4}}{\left(1-\left\|\overline{\boldsymbol{q}}_{\mathrm{r}}\right\|_{2}^{2}\right)^{2}}\right) \\
& =\left(1+\frac{J_{\mathbf{u}}\left(\underline{\boldsymbol{q}}_{\mathrm{r}}\right)}{\sigma_{s}^{2}}\right)^{-2}\left(1+\rho_{\max } \frac{J_{\mathbf{u}}^{2}\left(\underline{\boldsymbol{q}}_{\mathrm{r}}\right)}{\sigma_{s}^{4}}\right) .
\end{aligned}
$$

Thus, $1>\left(1+J_{\mathbf{u}}\left(\underline{\boldsymbol{q}}_{\mathrm{r}}\right) / \sigma_{s}^{2}\right)^{-2}\left(1+\rho_{\max } J_{\mathbf{u}}^{2}\left(\underline{\boldsymbol{q}}_{\mathrm{r}}\right) / \sigma_{s}^{4}\right)$ is sufficient for the left side of (31), which can be restated simply as $0<J_{\mathbf{u}}\left(\underline{\boldsymbol{q}}_{\mathrm{r}}\right) / \sigma_{s}^{2}<2\left(\rho_{\max }-1\right)^{-1}$. However, using the fact that $\rho_{\max } \geq 1$, it can be shown that $-1+2 \sqrt{\left(1+\rho_{\max }\right)^{-1}} \leq$ $2\left(\rho_{\max }-1\right)^{-1}$. Thus, $0<J_{\mathbf{u}}\left(\underline{\boldsymbol{q}}_{\mathrm{r}}\right)$ and (32) are sufficient for the two inequalities in (31).

For the case when $\rho_{\min }<0$ and $\mathcal{K}_{\mathrm{r}} / \mathcal{K}_{s}^{(0)}>\left(1+\rho_{\max }\right) / 4$, we know that

$$
\begin{aligned}
\rho_{\mathrm{r}} & \geq 1-2\left\|\overline{\boldsymbol{q}}_{\mathrm{r}}\right\|_{2}^{2}+\left(1+\rho_{\min }\right)\left\|\overline{\boldsymbol{q}}_{\mathrm{r}}\right\|_{2}^{4} \\
& =\left(1+\frac{J_{\mathbf{u}}\left(\underline{\boldsymbol{q}}_{\mathrm{r}}\right)}{\sigma_{s}^{2}}\right)^{-2}\left(1+\rho_{\min } \frac{J_{\mathbf{u}}^{2}\left(\underline{\boldsymbol{q}}_{\mathrm{r}}\right)}{\sigma_{s}^{4}}\right)
\end{aligned}
$$

$$
\rho_{\mathrm{r}}=\left|\frac{\mathcal{K}_{\mathrm{r}}}{\mathcal{K}_{s}^{(0)}}\right| \geq \begin{cases}1-2\left\|\overline{\boldsymbol{q}}_{\mathrm{r}}\right\|_{2}^{2}+\left\|\overline{\boldsymbol{q}}_{\mathrm{r}}\right\|_{2}^{4}, & \rho_{\min } \geq 0, \\ 1-2\left\|\overline{\boldsymbol{q}}_{\mathrm{r}}\right\|_{2}^{2}+\left(1+\rho_{\min }\right)\left\|\overline{\boldsymbol{q}}_{\mathrm{r}}\right\|_{2}^{4}, & \rho_{\min }<0 \text { and } \frac{\mathcal{K}_{\mathrm{r}}}{\mathcal{K}_{s}^{(0)}}>\frac{1+\rho_{\max }}{4} .\end{cases}
$$


which implies the bound in (33), shown at the bottom of the page. When $\rho_{\min }<0$, sufficient conditions for $\mathcal{K}_{\mathrm{r}} / \mathcal{K}_{s}^{(0)}>$ $\left(1+\rho_{\max }\right) / 4$ are

$$
\begin{aligned}
& 1-2\left\|\overline{\boldsymbol{q}}_{\mathrm{r}}\right\|_{2}^{2}+\left(1+\rho_{\min }\right)\left\|\overline{\boldsymbol{q}}_{\mathrm{r}}\right\|_{2}^{4}>\frac{1+\rho_{\max }}{4} \\
& \Leftrightarrow\left(1+\rho_{\min }\right)\left\|\overline{\boldsymbol{q}}_{\mathrm{r}}\right\|_{2}^{4}-2\left\|\overline{\boldsymbol{q}}_{\mathrm{r}}\right\|_{2}^{2}+\frac{3-\rho_{\max }}{4}>0 .
\end{aligned}
$$

It can be shown that the quadratic inequality above is satisfied by

$$
\left\|\overline{\boldsymbol{q}}_{\mathrm{r}}\right\|_{2}^{2}< \begin{cases}\frac{1-\sqrt{1-\frac{\left(3-\rho_{\max }\right)\left(1+\rho_{\min }\right)}{4}}}{1+\rho_{\min }}, & \rho_{\min } \neq-1 \\ \frac{3-\rho_{\max }}{8}, & \rho_{\min }=-1\end{cases}
$$

and since $J_{\mathbf{u}}(\underline{\boldsymbol{q}}) / \sigma_{s}^{2}=\|\overline{\boldsymbol{q}}\|_{2}^{2} /\left(1-\|\overline{\boldsymbol{q}}\|_{2}^{2}\right)$ is strictly increasing in $\|\overline{\boldsymbol{q}}\|_{2}^{2}$, the following must also be sufficient:

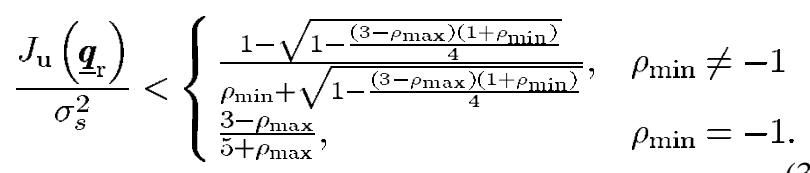

Note that $\mathcal{K}_{\mathrm{r}} / \mathcal{K}_{s}^{(0)}>\left(1+\rho_{\max }\right) / 4$, and thus, (34) ensures the right half of the existence condition (31). For the left half of (31), we have the same sufficient condition as when $\rho_{\min } \geq 0$. Thus, $0<J_{\mathbf{u}}\left(\underline{\boldsymbol{q}}_{\mathrm{r}}\right)$ and (34) are sufficient for the two inequalities in (31).

These results are summarized in Theorem 2 under the choice $\underline{\boldsymbol{q}}_{\mathrm{r}}=\underline{\boldsymbol{q}}_{\mathrm{m}} /\left\|\underline{\boldsymbol{q}}_{\mathrm{m}}\right\|_{2}$. Note that the UMSE conditions above guarantee that $\underline{q}_{\mathrm{m}} \in \mathcal{C}_{\nu}^{(0)}$, so we need not state it explicitly.

\section{Proof of Theorem 3}

Here, we reformulate the upper bound (22). To simplify the presentation of the proof, the shorthand notation $J:=J_{\mathrm{u}}\left(\underline{\boldsymbol{q}}_{\mathrm{m}}\right) / \sigma_{s}^{2}$ will be used.

Starting with the case $\rho_{\min } \geq 0$, (22) says

$$
\begin{aligned}
& \frac{\left.J_{\mathrm{u}}\right|_{\mathrm{sw}} ^{\max , J_{\mathrm{u}}}\left(\underline{\underline{q}}_{\mathrm{m}}\right)}{\sigma_{s}^{2}} \\
& =\frac{1-\sqrt{\left(1-\rho_{\max }\right)(1+J)^{-2}-\rho_{\max }}}{\rho_{\max }+\sqrt{\left(1+\rho_{\max }\right)(1+J)^{-2}-\rho_{\max }}}
\end{aligned}
$$

from which routine manipulations yield

$$
\begin{aligned}
\frac{\left.J_{\mathrm{u}}\right|_{\mathrm{sw}} ^{\max , J_{\mathrm{u}}}\left(\underline{\underline{q}}_{\mathrm{n}}\right)}{\sigma_{s}^{2}} \\
=\frac{1-\sqrt{1-\left(\left(\rho_{\max }-1\right)+\rho_{\max }\left(2 J+J^{2}\right)\right)\left(2 J+J^{2}\right)}}{\left(\rho_{\max }-1\right)+\rho_{\max }\left(2 J+J^{2}\right)} .
\end{aligned}
$$

For $x \in \mathbb{R}$ such that $|x|<1$, the binomial series [11] may be used to claim

$$
\sqrt{1-x}=1-\frac{x}{2}-\frac{x^{2}}{8}-\mathcal{O}\left(x^{3}\right)
$$

Applying the previous expression with $x=\left(\left(\rho_{\max }-1\right)+\right.$ $\left.\rho_{\max }\left(2 J+J^{2}\right)\right)\left(2 J+J^{2}\right)$, we find that

$$
\begin{aligned}
\frac{\left.J_{\mathbf{u}}\right|_{\mathrm{sw}} ^{\max }, J_{\mathrm{u}}\left(\underline{q}_{\mathrm{m}}\right)}{\sigma_{s}^{2}} & \\
= & \frac{1}{2} \frac{\left(\left(\rho_{\max }-1\right)+\rho_{\max }\left(2 J+J^{2}\right)\right)\left(2 J+J^{2}\right)}{\left(\rho_{\max }-1\right)+\rho_{\max }\left(2 J+J^{2}\right)} \\
& +\frac{1}{8} \frac{\left(\left(\rho_{\max }-1\right)+\rho_{\max }\left(2 J+J^{2}\right)\right)^{2}\left(2 J+J^{2}\right)^{2}}{\left(\rho_{\max }-1\right)+\rho_{\max }\left(2 J+J^{2}\right)} \\
& +\mathcal{O}\left(J^{3}\right) \\
= & J+\frac{\rho_{\max }}{2} J^{2}+\mathcal{O}\left(J^{3}\right) .
\end{aligned}
$$

Finally, subtraction of $J$ gives the first case in (23).

For the case $\rho_{\min }<0$, (22) says

$$
\begin{aligned}
& \frac{\left.J_{\mathrm{u}}\right|_{\mathrm{sw}} ^{\max , J_{\mathrm{u}}}\left(\underline{\underline{q}}_{\mathrm{m}}\right)}{\sigma_{s}^{2}} \\
& =\frac{1-\sqrt{\left(1+\rho_{\max }\right)(1+J)^{-2}\left(1+\rho_{\min } J^{2}\right)-\rho_{\max }}}{\rho_{\max }+\sqrt{\left(1+\rho_{\max }\right)(1+J)^{-2}\left(1+\rho_{\min } J^{2}\right)-\rho_{\max }}}
\end{aligned}
$$

from which routine manipulations yield the equation at the bottom of the page. As before, we use the binomial series expansion for $\sqrt{1-x}$ but now with

$$
\frac{\left.J_{\mathrm{u}}\right|_{\mathrm{sw}_{\mathrm{w}}} ^{\max , \mathcal{K}_{\mathrm{r}}}}{\sigma_{s}^{2}} \leq \frac{1-\sqrt{\left(1+\rho_{\max }\right)\left(1+\frac{J_{\mathrm{u}}\left(\underline{\boldsymbol{q}}_{\mathrm{r}}\right)}{\sigma_{s}^{2}}\right)^{-2}\left(1+\rho_{\min } \frac{J_{\mathrm{u}}^{2}\left(\underline{\underline{q}}_{\mathrm{r}}\right)}{\sigma_{s}^{4}}\right)-\rho_{\max }}}{\rho_{\max }+\sqrt{\left(1+\rho_{\max }\right)\left(1+\frac{J_{\mathrm{u}}\left(\underline{\underline{q}}_{\mathrm{r}}\right)}{\sigma_{s}^{2}}\right)^{-2}\left(1+\rho_{\min } \frac{J_{\mathrm{u}}^{2}\left(\underline{\underline{q}}_{\mathrm{r}}\right)}{\sigma_{s}^{4}}\right)-\rho_{\max }}} .
$$

$$
\frac{\left.J_{\mathrm{u}}\right|_{\mathrm{sw}} ^{\max , J_{\mathrm{u}}}\left(\underline{\boldsymbol{q}}_{\mathrm{m}}\right)}{\sigma_{s}^{2}}=\frac{\rho_{\min } J^{2}+1-\sqrt{1+\left(2-2 \rho_{\min }\right) J+\left(1+\rho_{\min }+\rho_{\max } \rho_{\min }-5 \rho_{\max }\right) J^{2}+\mathcal{O}\left(J^{3}\right)}}{\left(\rho_{\max }-1\right)+2 \rho_{\max } J+\left(\rho_{\max }-\rho_{\min }\right) J^{2}} .
$$


$x=\left(2 \rho_{\min }-2\right) J+\left(5 \rho_{\max }-1-\rho_{\min }-\rho_{\max } \rho_{\min }\right) J^{2}+\mathcal{O}\left(J^{3}\right)$.

After some algebra, we find

$$
\begin{aligned}
& \frac{\left.J_{\mathbf{u}}\right|_{\mathrm{sw}} ^{\max , J_{\mathrm{u}}\left(\underline{q}_{\mathrm{n}}\right)}}{\sigma_{s}^{2}} \\
& \quad=J+\frac{1}{2} \frac{\left(\rho_{\max }-\rho_{\min }\right)\left(\rho_{\max }-1\right) J^{2}+\mathcal{O}\left(J^{3}\right)}{\left(\rho_{\max }-1\right)+2 \rho_{\max } J+\left(\rho_{\max }-\rho_{\min }\right) J^{2}} .
\end{aligned}
$$

Finally, we apply the series approximation $(1-y)^{-1}=1+y+$ $\mathcal{O}\left(y^{2}\right)$ with $y=-\left(2 \rho_{\max } J+\left(\rho_{\max }-\rho_{\min }\right) J^{2}\right) /\left(\rho_{\max }-1\right)$ for $\rho_{\max } \neq 1$. Straightforward algebra yields

$$
\frac{\left.J_{\mathbf{u}}\right|_{\mathrm{sw}} ^{\max , J_{\mathrm{u}}\left(\underline{\boldsymbol{q}}_{\mathrm{m}}\right)}}{\sigma_{s}^{2}}=J+\frac{1}{2}\left(\rho_{\max }-\rho_{\min }\right) J^{2}+\mathcal{O}\left(J^{3}\right) .
$$

Taking the limit $\rho_{\max } \rightarrow 1$, it is evident that no problems arise at the point $\rho_{\max }=1$. Subtraction of $J$ from the last statement gives the second case in (23).

\section{ACKNOWLEDGMENT}

The authors would like to sincerely thank P. Regalia for his many helpful comments and suggestions.

\section{REFERENCES}

[1] O. Shalvi and E. Weinstein, "New criteria for blind deconvolution of nonminimum phase systems (channels)," IEEE Trans. Inform. Theory, vol. 36, pp. 312-321, Mar. 1990.

[2] H. F. Kaiser, "The varimax criterion for analytic rotation in factor analysis," Psychometrica, vol. 23, no. 3, pp. 187-200, Sept. 1958.

[3] R. A. Wiggins, "Minimum entropy deconvolution," Geoexploration, vol. 16 , pp. 21-35, 1978

[4] D. L. Donoho, "On minimum entropy deconvolution," in Applied Time Series Analysis II, D. Findley, Ed. New York: Academic, 1981, pp. 565-608.

[5] P. Regalia and M. Mboup, "Undermodeled equalization: A characterization of stationary points for a family of blind criteria," IEEE Trans. Signal Processing, vol. 47, pp. 760-70, Mar. 1999.

[6] C. C. Feng and C. Y. Chi, "Performance of cumulant based inverse filters for blind deconvolution," IEEE Trans. Signal Processing, vol. 47, pp. 1922-35, July 1999.
[7] _ - "Performance of Shalvi and Weinstein's deconvolution criteria forchannels with/without zeros on the unit circle," IEEE Trans. Signal Processing, vol. 48, pp. 571-5, Feb. 2000.

[8] P. Schniter, "Blind estimation without priors: Performance, convergence, and efficient implementation," Ph.D. dissertation, Cornell Univ., Ithaca, NY, 2000.

[9] B. Porat, Digital Processing of Random Signals. Englewood Cliffs, NJ: Prentice-Hall, 1994.

[10] D. G. Luenberger, Optimization by Vector Space Methods. New York: Wiley, 1968.

[11] W. Rudin, Principles of Mathematical Analysis, 3rd ed. New York: McGraw-Hill, 1976.

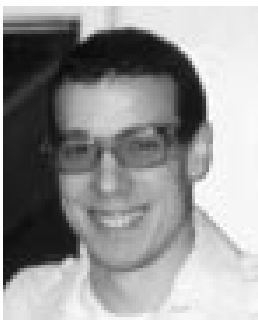

Philip Schniter (M'00) was born in Evanston, IL, in 1970. He received the B.S. and M.S. degrees in electrical and computer engineering from the University of Illinois, Urbana-Champaign, in 1992 and 1993, respectively. In 2000, he received the Ph.D. degree in electical engineering from Cornell University, Ithaca, NY.

From 1993 to 1996, he was employed by Tektronix Inc., Beaverton, OR, as a systems engineer. There, he worked on signal processing aspects of video and communications instrumentation design, including algorithms, software, and hardware architectures. He is currently an Assistant Professor with the Department of Electrical Engineering, The Ohio State University, Columbus. His research interest is signal processing for communication systems, especially blind adaptive equalization.

While pursuing the $\mathrm{Ph} . \mathrm{D}$. degree, he received the 1998 Schlumberger Fellowship and the 1998-1999 Intel Foundation Fellowship. He received the 1999 Prize Paper Award from the IEEE Energy Development and Power Generation Committee for work relating to his M.S. thesis.

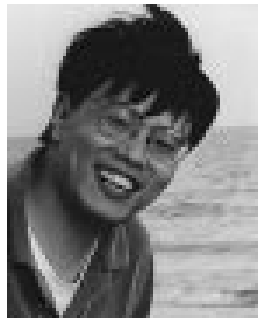

Lang Tong (S'87-M'91) received the B.E. degree from Tsinghua University, Beijing, China, in 1985 and the M.S. and Ph.D. degrees in electrical engineering in 1987 and 1990, respectively, from the University of Notre Dame, Notre Dame, IN.

He was a Postdoctoral Research Affiliate with the Information Systems Laboratory, Stanford University, Stanford, CA, in 1991. Currently, he is an Associate Professor with the School of Electrical and Computer Engineering, Cornell University, Ithaca, NY. His areas of interest include statistical signal processing, adaptive receiver design for communication systems, signal processing for communication networks, and information theory.

Dr. Tong received the Young Investigator Award from the Office of Naval Research in 1996 and the Outstanding Young Author Award from the IEEE Circuits and Systems Society. 